\title{
Recent progress in optoelectronic applications of hybrid 2D/3D silicon- based heterostructures
}

\author{
Jingshu Zhou ${ }^{1,2}$, Kaiyao Xin ${ }^{1}$, Xiangkai Zhao ${ }^{3 *}$, Dongmei $\mathrm{Li}^{3}$, Zhongming Wei $^{{ }^{*}}$ and Jianbai Xia ${ }^{1}$
}

\begin{abstract}
Silicon-based semiconductor technology has made great breakthroughs in the past few decades, but it is reaching the physical limits of Moore's law. In recent years, the presence of two-dimensional (2D) materials was regarded as an opportunity to break the limitation of traditional siliconbased optoelectronic devices owing to their special structure and superior properties. In consideration of the widely studied hybrid integration of $2 \mathrm{D}$ material detectors and $3 \mathrm{D}$ siliconbased systems, in this paper, the basic properties of several 2D materials used in photodetectors are summarized. Subsequently, the progress in silicon photonic integrated photodetectors based on 2D materials is reviewed, followed by the summarization of the device structure and main performances. Then, the combination of some other traditional and 2D devices is discussed as a supplement. Finally, the prospective development of the hybrid 2D/3D silicon-based heterostructures is expected.
\end{abstract}

Keywords: silicon, 2D/3D, hybrid heterostructure, optoelectronic application

\section{INTRODUCTION}

In the middle of last century, low-dimensional materials had entered the scientists' sight. After the experimental demonstration of graphene realized by Novoselov and Geim [1], the exploration of low-dimensional materials stepped into an explosive stage. In addition to in-depth investigations on graphene, not only a large number of new low-dimensional materials have been discovered, but the old bulk materials have been injected with new vitality. Research in recent years has shown that low-dimensional materials possess many unprecedented merits, such as strong light-matter coupling [2], adjustable bandgap [3], high carrier mobility [4], low dark current [5], appreciable signal-to-noise ratio [6], ultra-low energy dissipation [7], and fast response/recover speed [8]. These advantages have made low-dimensional materials potential to outperform traditional 3D bulk materials in the next generation of optoelectronics. Therefore, it is of great promise for low-dimensional materials to serve in diverse applications especially optoelectronic fields with outstanding performance.

Two-dimensional (2D) material-based devices have been widely used in photovoltaics, semiconductor, electrode and biological monitoring applications due to their unique proper- ties. At the same time, there is less exploitation space left for conventional substrates, including $\mathrm{Si}[9,10], \mathrm{Ge}[11,12], \mathrm{GaN}$ [13], InP [14], and mica [15], since the mature industrial technology and natural unlayered crystal structures. 2D materials, while unlikely to replace the conventional materials, can complement existing technologies. Microelectronic devices will continue to develop in the direction of faster, smaller and cheaper. Graphene, transitional metal dichalcogenides (TMDCs) and other new 2D materials, with unique optical, electrical and magnetic properties and new quantum physical phenomena, have potential applications in information, micro-nano optoelectronics and other aspects. So, the cross-dimensional heterostructures which could combine the advantages of both lowdimensional materials and conventional substrates will be the best choice in the future. To date, various hybrid $1 \mathrm{D} / 2 \mathrm{D}[16,17]$, $2 \mathrm{D} / 2 \mathrm{D}[18,19]$, and 2D/3D $[20,21]$ heterostructure devices have been successfully fabricated. The $2 \mathrm{D} / 3 \mathrm{D}$ heterojunction photodetectors have attracted the most attention, which has been considered as the most feasible scheme. Because on one hand, the light absorption efficiency in 2D/3D heterostructures would be much stronger than in ultrathin $1 \mathrm{D}$ (2D)/2D stacking, laying solid foundation for generating photocarriers. On the one hand, the $2 \mathrm{D} / 3 \mathrm{D}$ heterostructure devices could exhibit excellent properties that $2 \mathrm{D}$ devices and $3 \mathrm{D}$ devices cannot show alone. On the other hand, 2D/3D heterojunction photodetectors can be easily achieved by directly integrating $2 \mathrm{D}$-layered materials onto mature 3D commercial substrates such as $\mathrm{Si}$ and $\mathrm{GaAs}$ via interlayer van der Waals interaction. The van der Waals gap in $2 \mathrm{D}$ materials has the potential to accommodate intercalated atomic and molecular species. In fact, epitaxial graphene on $\mathrm{SiC}$ has been a model system for studying intercalation, and a decoupling graphene/SiC buffer layer typically causes charge carrier scattering and n-type doping in epitaxial graphene [22]. For example, annealing epitaxial graphene at high temperatures in a $\mathrm{H}_{2}$ environment promotes hydrogen intercalation between the buffer layer and the SiC substrate, resulting in passivation of the Si dangling bonds. Among diverse traditional platforms, optoelectronics based on silicon substrate are compatible with complementary metal-oxide-semiconductor (CMOS) processing, which can achieve mass production with the help of mature microelectronics technology. In addition, there are many advantages of silicon wafers, including low cost, easy accessibility, reliability and ability to achieve high integration. Hence, it is expectable as well as promising to realize the integration of

\footnotetext{
${ }^{1}$ State Key Laboratory of Superlattices and Microstructures, Institute of Semiconductors, Chinese Academy of Sciences, Beijing 100083, China

2 School of Electronic, Electrical and Communication Engineering, University of Chinese Academy of Sciences, Beijing 100049, China

${ }^{3}$ Optoelectronic System Laboratory, Institute of Semiconductors, Chinese Academy of Sciences, Beijing 10083, China

* Corresponding authors (emails: zmwei@semi.ac.cn (Wei Z); zhao_xiangkai@semi.ac.cn (Zhao X))
} 
low-dimensional materials on silicon-based substrates. Until now, intensive efforts have been devoted to low-dimensional material/Si heterojunction devices. These silicon-based heterostructural devices have been applied in many fields, such as photodetecting, infrared imaging, polarization monitoring, and flexible device producing.

It is generally accepted that low-dimensional material/Si heterostructures are also easy to fabricate. For example, by transferring graphene on $\mathrm{Si}$, An et al. [23] obtained an ultrasensitive graphene/Si heterojunction photodetector and realized a high responsivity of $0.33 \mathrm{~A} \mathrm{~W}^{-1}$. For another example, by directly growing $\mathrm{MoS}_{2}$ on Si substrate, Wang et al. [24] fabricated a $\mathrm{MoS}_{2} / \mathrm{Si}$ heterojunction. Fig. 1 shows the schematic structure of the Si-based cross heterostructures based on different types of $2 \mathrm{D}$ materials. Herein, this review will be focused on the Si-based $2 \mathrm{D} / 3 \mathrm{D}$ heterostructures to display the properties of crossdimensional devices, along with some low-dimensional materials as complement.

\section{PREPARATION METHODS}

In general, there are two strategies in the preparation of lowdimensional materials, top-down and bottom-up. The top-down method means a reduction of thickness from the bulk material to monolayer or few layers such as exfoliation. The main approaches of the top-down method are mechanical exfoliation (ME) and mechanically assisted exfoliation. ME that helped Novoselov et al. [1] to discover graphene is the easiest way to get low-dimensional materials, and it was the milestone for the fabrication of low-dimensional materials. For the case of bottom-up method, chemical vapor deposition (CVD) is one of the most widely used methods for that high-quality low-dimensional materials can be controllably grown. Meanwhile, molecular beam epitaxy (MBE) and other epitaxial technologies are also usually used to synthesize low-dimensional materials. Subse- quently, a brief introduction of these common methods will be described in this section.

\section{Mechanical exfoliation}

The ME was first created and utilized by Novoselov et al. [1]. They got few-layer graphene by repeated peeling from bulk graphite on transparent tapes, which ignited the flame of investigation on $2 \mathrm{D}$ materials and provided a cheap, easy but feasible way to access. Even now, the ME is a common means for researchers to get other kinds of low-dimensional materials.

Many assistant tools were designed to help exfoliate lowdimensional materials. Sonication-assisted exfoliation and shearforce-assisted liquid phase exfoliation (LPE) [26], ball millingassisted exfoliation [27] are typical and commonly used in mechanically assisted exfoliation. The main principle of LPE is to take the bulk material as the raw chemical, adding to a specific solvent or surfactant to form a pretreatment solution. Then the monolayer or few-layer material from the bulk surface will be peeled with the help of high-power ultrasonic equipment. The LPE method takes advantage of cavitation and high shear force from the ultrasonic wave. The cavitation makes the bulk material be crushed, while the shear force makes the solvent form a micro-jet impact, which promotes the separation between the layers of the bulk material. Early in 2008, Coleman's group [28] added graphite powders into organic solvents, a mixed solution of $N, N$-dimethylformamide and $N$-methylpyrrolidone, and then treated the mixture by means of ultrasound and centrifugation, obtaining the graphene with few defects at last, and the production efficiency of graphene was nearly $0.01 \mathrm{mg} \mathrm{mL}^{-1}$ after $30 \mathrm{~min}$ ultrasonic treatment. LPE features easy operation and high production efficiency, so that it is expected to be a potential path in industrial production of low-dimensional materials, especially graphene. On the other hand, LPE can be used to obtain some few-layer or single-layer materials that are difficult

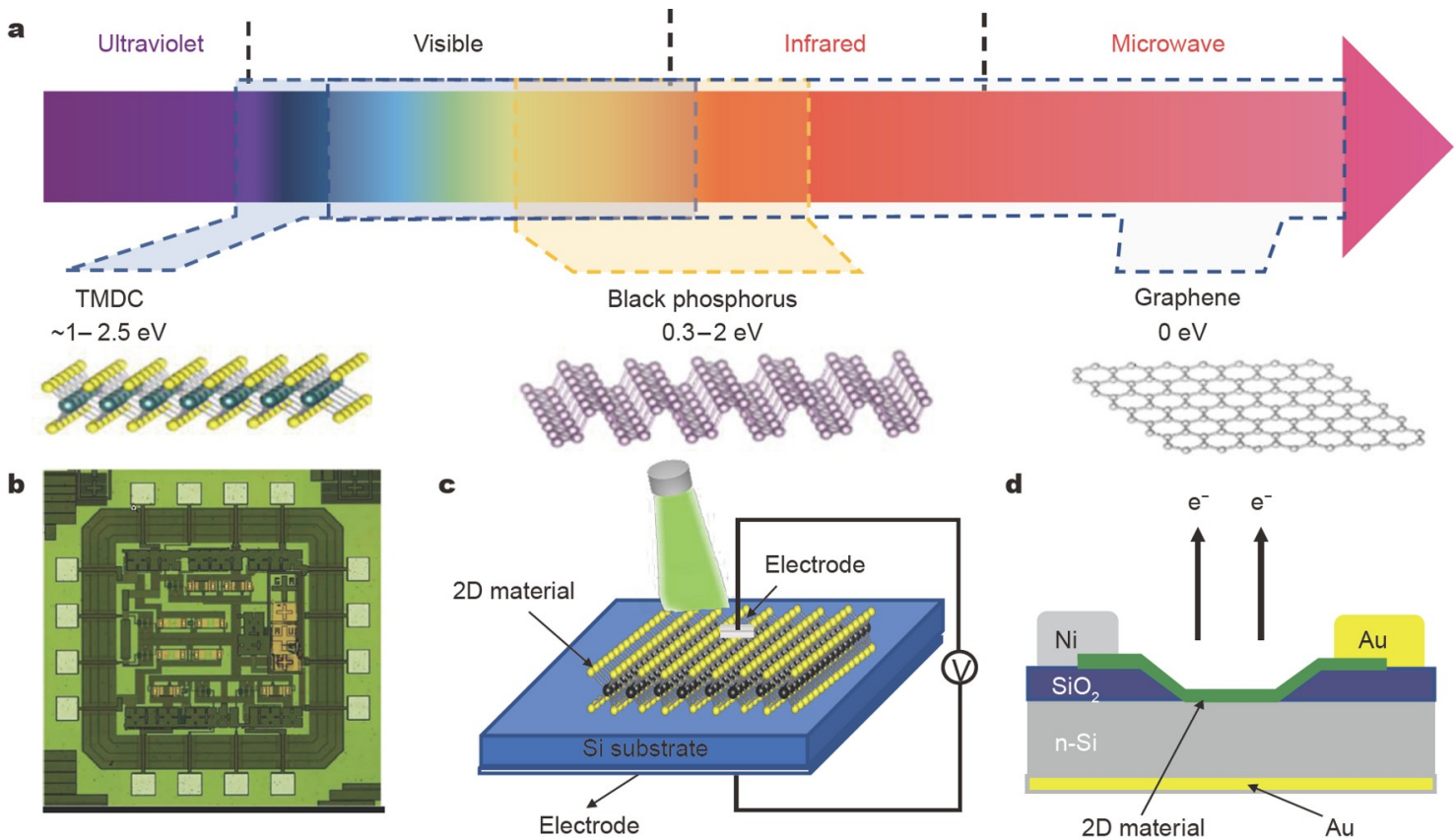

Figure 1 (a) Diverse types of 2D materials and their response ranges. (b) Optical image of 2D materials/Si heterostructures. The 2D materials are combined with Si substrate and the top electrode is attached to the oxide layer. (c) Schematic diagram of a 2D/Si heterostructure device. (d) Sectional view of a $2 \mathrm{D} / \mathrm{Si}$ optoelectronic device. (b) is reprinted with permission from Ref. [25]. Copyright 2021, Springer Nature Limited. 
to peel by ME. Besides, many other famous $2 \mathrm{D}$ materials, including h-BN [29], some kinds of TMDCs [30], and black phosphorus (BP) [31], could also be obtained by LPE. To enhance the efficiency and increase the production efficiency of low-dimensional materials, tip sonication with a high power density and high shear rates could be used. Ball-milling-assisted exfoliation, adept at producing large-scale samples, is another mechanical method to exfoliate low-dimensional materials, which also takes advantage of shear force. And several materials (graphene [32], h-BN [33], $\mathrm{MoS}_{2}$ [34]) have been successfully obtained by using this method.

\section{Epitaxial methods}

Although relatively high-quality materials could be obtained through aforementioned approaches, the acquisition of the products is almost uncontrollable and the final size of the crystal is restrained. In order to get large-scale or even wafer-scale monolayer, it is generally accepted that epitaxial growth will be the most appropriate as well as feasible way. Because compared with the exfoliation, the nucleation and growth processes in epitaxial growth are under precise control, resulting in accurate arrangement of the thickness and atomic configuration, which acts as the fundamental for obtaining large-scale products. In this part, we will introduce some epitaxial methods to prepare low-dimensional materials.

\section{Chemical vapor deposition}

CVD is the most popular technique for preparing high-purity and high-performance films. Some heterostructures can be formed by growing low-dimensional materials directly on the substrate via CVD technology $[35,36]$. In the process of CVD, one or more vapor source atoms or molecules are carried by carrier gas in the chamber, and the desired film will be formed on the substrate surface through chemical reaction. CVD is usually used to grow graphene, h-BN, $\mathrm{MX}_{2}(\mathrm{M}=\mathrm{Mo}, \mathrm{W}$, Re and so on, $\mathrm{X}=\mathrm{S}, \mathrm{Se}, \mathrm{Te})$, and some kinds of metal oxides. In the CVD process, the dosage ratio of raw materials plays a critical role. For example, Zhou et al. [37] demonstrated an effective CVD strategy, using a Te:metal oxide:metal chloride weight ratio of $1: 1: 1$, to synthesize few-layer and monolayer $\mathrm{WTe}_{2}$ and $\mathrm{MoTe}_{2}$ on a large scale [38]. However, it is not generally suitable for all low-dimensional materials. For example, BP, b- $\mathrm{As}_{x} \mathrm{P}_{1-x}$ and metal oxides cannot be obtained through CVD, requiring complementary fabrication methods by which the shortcomings of CVD could be overcome.

\section{Molecular beam epitaxy}

MBE is a technology to epitaxially grow materials on the crystalline substrate in an ultrahigh-vacuum environment. MBE can precisely control the thickness, purity, doping density and composition of dopants. Nevertheless, the popularization of MBE in fabrication of 2D materials is limited by its high cost and complex operation [39]. To satisfy different demands of various materials, other epitaxial methods are exploited. For example, considering the difficulty of preparing large-scale as well as atomically thin BP film by ME and CVD, Xu et al. [40] reported the direct growth of large-scale crystalline $\mathrm{BP}$ films on insulating silicon substrates. With polyphosphide $\mathrm{Au}_{3} \mathrm{SnP}_{7}$ serving as the nucleation seeds, large-size BP film appeared from epitaxial nucleation to controllable lateral growth, following a newly developed gas phase growing strategy. As reported, the field- effect and Hall mobility reached 1000 and $1400 \mathrm{~cm}^{2} \mathrm{~V}^{-1} \mathrm{~s}^{-1}$ at room temperature, respectively.

\section{Atomic layer deposition}

In low-dimensional heterostructures, insulating layer would usually be introduced to make a potential barrier. Atomic layer deposition (ALD) is a special CVD technique used to deposit atomically thin films onto a substrate. By ALD, controllable thickness, uniformity, composition of the film and compatibility to most substrates can be realized at the same time. Furthermore, ALD can be used to prepare ultrathin films of many materials, such as oxides, nitrides, sulfides, carbides, fluoride and even polymers $[41,42]$. It is noteworthy that the ALD technology is usually carried out in vacuum at relatively low temperatures, with the help of which ALD could be applied to sensitive surface but free of pinholes, cracks and defects. Some high-K oxide layers serving as dielectric layers, such as $\mathrm{Al}_{2} \mathrm{O}_{3}, \mathrm{HfO}_{2}$, and $\mathrm{SiO}_{2}$ $[43,44]$, are typically prepared by ALD.

\section{Electron beam evaporation}

Electron beam evaporation (EBE) method is a kind of vacuum evaporation coating method, which uses electron beam to evaporate materials by heat in vacuum environment [45]. The evaporated materials will be transported to the substrate and condensed into thin films on the substrate. In the electron beam heating device, the heated material is placed in the water-cooled orange vortex, which can avoid the reaction between the evaporating material and the orange wall, thus leading to final products with high purity and quality. Moreover, multiple crucibles can be installed in the same equipment to realize simultaneous or sequential evaporation and deposition. Even the materials with high melting point can be turned into highquality thin film by EBE, which makes it a widely popular method in preparation of conductive glass and other optical films.

\section{FIGURE OF MERITS}

Photodetectors are the devices in which electronic signals would be monitored after absorption of external photons. Researchers have put forward several kinds of mechanics to explain the photovoltaic conversion for various photodetectors. The most common mechanisms are photoconductive effect (PCE) and photovoltaic effect (PVE). When the photon energy of incident light is larger than the bandgap energy, the electrons will be transited to excited state, leading to the emergence of nonequilibrium carriers, thus increasing the total carrier concentration. In this case, an external electric field should be exerted to separate the photon-generated electron-hole pairs, by which the difference between photocurrent and dark-current could be distinguished. Particularly, photogating effect (PGE) could be seen as a special circumstance of PCE in the case of photodetectors based on ultrathin low-dimensional materials. It utilizes the trapped stage as a localized floating gate to modulate the channel conductance. On the contrary, for the PVE photodetectors, the photogenerated carrier will be separated by a builtin electric field, and the photocurrent could be formed without extra power. Moreover, photothermoelectric effect (PTE) and bolometric effect (BE) are usually used to explain the photoelectric process which is dominated by thermodynamics principle.

Although it seems that various photodetectors work following 
different mechanisms, there are some universal standards to estimate the quality of these photodetectors. In the following part, we will introduce some primary indicators.

\section{Responsivity $(R)$}

Responsivity represents the ability of photoelectric conversion of photodetectors, which is defined as the ratio of the generated photocurrent $\left(I_{\mathrm{ph}}\right)$ or output voltage $\left(V_{\mathrm{ph}}\right)$ to the average power of the incident light $\left(P_{\text {in }}\right)$ on the photodetector. It can be calculated by the following equation:

$R_{\mathrm{i}}=\frac{I_{\mathrm{ph}}}{P_{\text {in }} S}$, or $R_{\mathrm{v}}=\frac{V_{\mathrm{ph}}}{P_{\text {in }} S}$,

where $S$ is the illuminated area, the units of the $R_{\mathrm{i}}$ (responsivity based on photocurrent) and $R_{\mathrm{v}}$ (responsivity based on voltage) are $\mathrm{A} \mathrm{W}^{-1}$ and $\mathrm{V} \mathrm{W}^{-1}$, respectively.

\section{External quantum efficiency (EQE)}

It is well-known that when incident light is shined on the device, not all photons will be used to generate nonequilibrium carriers. EQE denotes the ability for a photodetector to transform the photonic signal into an electrical signal, which is calculated as the number of electron/hole pairs detected per incident photon, given by the following equation:

$\mathrm{EQE}=R h c / e \lambda$,

where $h$ is Planck's constant, $c$ is the light velocity, $e$ is the electron charge and $\lambda$ is the excitation wavelength. In some situations, the value of EQE may be larger than 1 because the photo-generated carrier lifetime is longer than the transit time, and it will be calculated several times for one photo-generated carrier.

\section{Noise equivalent power (NEP) and detectivity $\left(D^{*}\right)$}

NEP is the minimum detectable power of a photodetector and $D^{*}$ is inversely proportional to NEP. Both $D^{*}$ and NEP represent the detection ability of a photodetector. In general, people prefer detectivity than NEP to express the property of a detector, which can be given by:

$D^{*}=\frac{A^{1 / 2} R}{\left(2 e I_{\mathrm{d}}\right)^{1 / 2}}$

where $A$ is the devive area. In this equation, we can see a low dark current $\left(I_{\mathrm{d}}\right)$ and high responsivity will help to achieve a larger $D^{*}$

\section{Photoconductive gain $(G)$}

For photodetectors, the photocurrent originates from the detected photons, so the photoconductive gain is described by:

$G=\frac{\tau_{\text {life }}}{\tau_{\text {trans }}}$,

where $\tau_{\text {life }}$ is the carrier's lifetime, and $\tau_{\text {trans }}$ is carrier's drift time. From the equation, we can see the $G$ implies the number of carriers that a single incident photon excites. Long carrier life means high gain, but also longer response time.

\section{On/off ratio}

On/off is defined as the ratio of the open state current $\left(I_{\text {on }}\right)$ to the off state current $\left(I_{\text {off }}\right)$ of the device. For an ideal switch, the off current will be zero and the on current will only be limited by the external circuit.

\section{DEVICES BASED ON DIFFERENT 2D MATERIALS}

To date, devices based on various low-dimensional materials have been fabricated including graphene, TMDCs, elemental semiconductors (like BP, Te), main group metal chalcogenides (MMCs), thin film of unlayered materials and some heterostructures. These nanomaterials could cover the wide range of electromagnetic spectrum, showing many novel properties that traditional devices do not have. The new performances have attracted scientists' eyesight, and the new generation devices may play an important role in the future electronic and optoelectronic field. In this section, we will introduce these wellperformed devices from the perspective of materials.

\section{Graphene-based materials}

Graphene is a single low-dimensional sheet of carbon atoms with a honeycomb lattice structure, in which its carbon atoms attach each other to form a hexagonal shape, and its layers combine via van der Waals interaction. The single-layer and few-layer graphene was firstly obtained by Novoselov et al. [1] via a scotch tape method, and then they took a closer look at the properties of graphene. As they reported, graphene has many good properties that not previously discovered in other semiconductor materials, such as zero bandgap, ultrahigh carrier mobility (about $10,000 \mathrm{~cm}^{2} \mathrm{~V}^{-1} \mathrm{~s}^{-1}$ at room temperature, $200,000 \mathrm{~cm}^{2} \mathrm{~V}^{-1} \mathrm{~s}^{-1}$ at low temperature), and high conductivity.

\section{The pristine graphene-based optoelectronic devices}

Due to its outstanding properties, quite a few scientists put a lot of effort into graphene, and among the vast number of lowdimensional materials, the studies of graphene are the most, which also have implication for other low-dimensional materials. In 2012, a graphene metal-oxide-semiconductor field-effect transistor (MOSFET) with an intrinsic cutoff frequency exceeding $400 \mathrm{GHz}$ was successfully demonstrated [46]. The encouraging development misled scientists, so that graphene had been designated as the successor of $\mathrm{Si}$ in mainstream electronics. However, as the research of graphene continues, the graphene/Si heterostructure-based devices can only attain an $I_{\text {on }} / I_{\text {off }}$ ratio of at most a few tens due to their zero bandgap, which limits the further application into integrated circuits. To solve this problem, scientists have designed many schemes to open a gap in graphene. Nevertheless, the as-made opened-gap graphenebased devices had worse performance than the primary ones. Therefore, to explore the graphene-based devices in a commercial use, there are a lot of problems to be solved in the nearto medium-term future.

A lot of graphene heterostructure-based photodetectors have been finished. Graphene-based optoelectronic devices present ultra-broadband photoresponse due to its zero bandgap. As early as in 2010 , the IBM group designed a graphene/Si heterostructure-based photodetector [47], whose response range covered 514 to $2400 \mathrm{~nm}$, and the as-made photodetector also had good performances with the maximum external photoresponsivity of $6.1 \mathrm{~mA} \mathrm{~W}^{-1}$ at a wavelength of $1.55 \mu \mathrm{m}$. In a recent research, $\mathrm{THz}$ graphene-based detectors have been fabricated and they have a potential to be used in outer space exploration. Lara-Avil et al. [48] synthesized graphene sheets that were uniformly doped to the Dirac point and then were used to construct graphene/Si heterostructure-based photodetectors. With material resistance dominated by quantum localization and thermal relaxation governed by electron diffu- 
sion, the photodetectors showed highly sensitive and wide-band coherent detection of signals from 90 to $700 \mathrm{GHz}$ and maybe across the entire terahertz range. Then researchers conducted $\mathrm{THz}$ mixing experiments, a gain bandwidth of $f_{0}=8 \mathrm{GHz}$ was demonstrated. And at a low temperature $(36 \mathrm{~K})$, the gain bandwidth would exceed $20 \mathrm{GHz}$. These breakthroughs in detector may accelerate the development of heterodyne astronomy. And for some photodetectors, graphene could be an excellent electrode material due to its high conductivity and transparency [49].

The improvements for graphene-based devices

For most graphene/Si heterostructure-based devices, they have high leakage current noise, relatively high dark currents and low light absorption, which limit the further development of highperformance photodetectors. To reduce the dark current, researchers have tried some ways, one of which is to introduce a small gap to suppress the dark current, for that a small gap functioned like a quantum well helps suppress the dark current. For example, Ryzhii et al. [50] designed several prototype devices to improve the performances of graphene-based photodetectors. The first design was based on bilayer graphene in which the energy gap can be changed by the external electric field with a maximum detectivity over $10^{11}$ Jones. The second one was based on tailored graphene nanoribbons [51] in which the band gap can be changed by controlling the width of nanoribbons with a responsivity of about $6.4 \mathrm{~A} \mathrm{~W}^{-1}$. Based on these two prototypes, they developed a variety of high-performance $\mathrm{THz}$ infrared graphene detectors in recent years $[52,53]$.
On the other hand, a $\mathrm{p}-\mathrm{i}-\mathrm{n}$ structure by introducing a thin interfacial insulative layer is a good way to improve the band structure of graphene/Si heterostructure. Li et al. [54] reported a design that introduces an interfacial oxide layer between the graphene and n-silicon; the dark current of graphene/n-Si heterojunction has been reduced by two orders of magnitude at zero bias. At room temperature, the graphene/n-Si photodetector with interfacial oxide exhibits a specific detectivity up to $5.77 \times 10^{13}$ Jones with a responsivity of $0.73 \mathrm{~A} \mathrm{~W}^{-1}$ as well as a high on/off ratio of $\sim 10^{7}$. Fig. $2 \mathrm{a}-\mathrm{d}$ show the $\mathrm{p}-\mathrm{i}-\mathrm{n}$ structure and its effect on dark current suppression. In a latest report [11], a thin layer of $\mathrm{HfO}_{2}$ was introduced in the graphene/Si heterostructure to enhance the interfacial gating effect. The as-made graphene $/ \mathrm{HfO}_{2} / \mathrm{Si}$ heterostructure photodetectors attained better performances with a broad response range from 473 to $1150 \mathrm{~nm}$, a high responsivity of $45.8 \mathrm{~A} \mathrm{~W}^{-1}$, fast response speed of $\tau_{\text {rise }}=$ $9 \mu \mathrm{s}, \tau_{\text {decay }}=20 \mu \mathrm{s}$. Fig. $2 \mathrm{e}$, f show the schematics and photoresponse characteristics of the graphene $/ \mathrm{HfO}_{2} / \mathrm{Si}$ photodetector.

Decorating metal plasmonic nanoparticles (arrays) on the surface of graphene is a good way to improve the photocurrent. The plasmonic nanoparticles could enhance the field on the surface of metal, leading to an effective improvement to the photo gain of the photodetectors. Ferrari and coworkers [55] fabricated a plasmonic-enhanced metal/single-layer graphene (SLG)/Si Schottky photodetector with a responsivity of $0.37 \mathrm{~A} \mathrm{~W}^{-1}$ (operating at $1.5 \mu \mathrm{m}$ ) and an internal quantum efficiency (IQE) of 7\% (see Fig. 3a, b). To improve the response speed, Selvi et al. [56] investigated a graphene-silicon-on-insulator (GSOI) Schottky junction. In this junction, the thickness of
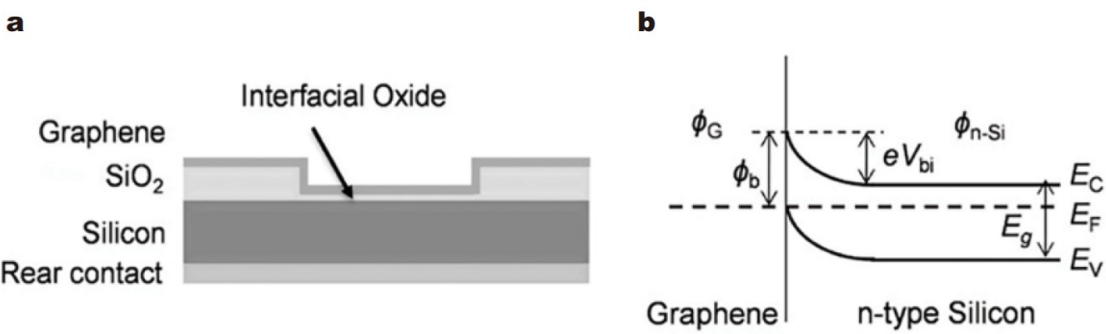

d

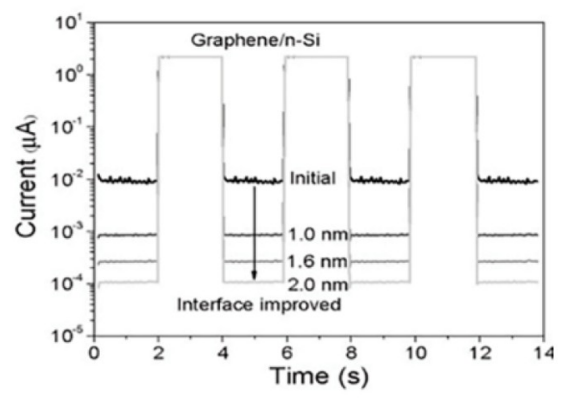

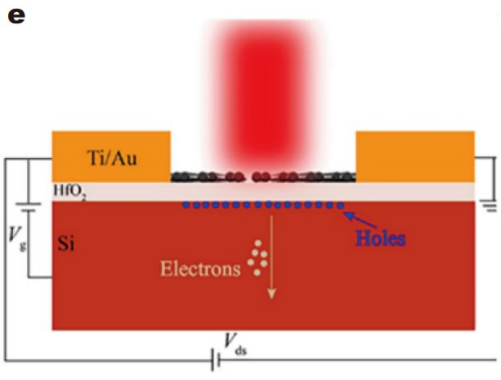
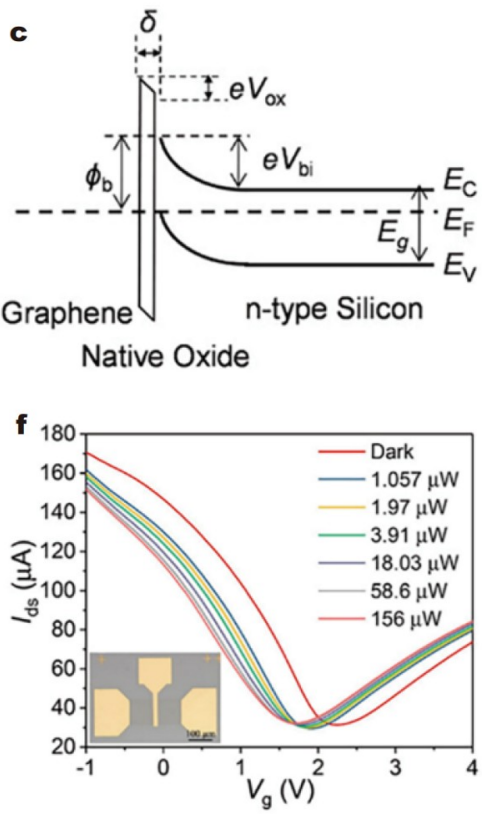

Figure $2 \mathrm{p}$-i-n structures to suppress dark current. (a) Schematic view of a graphene/n-Si heterojunction photodetector with an interfacial oxide layer. The silicon wafer with a 300-nm $\mathrm{SiO}_{2}$ layer was patterned by photolithography and wet-etching of thermally grown oxide to prepare square windows. The energyband diagrams of the heterojunction of (b) graphene/n-Si and (c) graphene $/ \mathrm{SiO}_{2} / \mathrm{Si}$. $\varphi_{\mathrm{G}}$ and $\varphi_{\mathrm{n}-\mathrm{Si}}$ are the work functions of graphene and n-Si, respectively; $\varphi_{\mathrm{b}}$ is the ideal barrier height. $V_{\mathrm{bi}}$ is the built-in potential barrier. $V_{\mathrm{ox}}$ is the potential drop across the oxide layer. $\delta$ is the thickness of oxide layer. $E_{\mathrm{g}}$ is the band gap of silicon $(1.12 \mathrm{eV})$ and $E_{\mathrm{F}}$ is the energy of the Fermi level. (d) Oxide-thickness-dependent response of the heterojunction photodetectors. (a-d) are reprinted with permission from Ref. [54]. Copyright 2015, Wiley-VCH Verlag GmbH \& Co. KGaA, Weinheim. (e) Schematic view of the graphene/HfO $/ 2$ Si photodetector with a pair of 10/50 nm Ti/Au source-drain electrodes. Incident photons create electron-hole pairs in Si. These photogenerated carriers are separated by the built-in field and the accumulation of the holes at the $\mathrm{HfO}_{2} / \mathrm{Si}$ interface gates the graphene through capacitive coupling. (f) Transfer curves of the photodetector at various light powers. The inset is the optical image of the photodetector. (e, f) are reprinted with permission from Ref. [11]. Copyright 2021, American Chemical Society. 

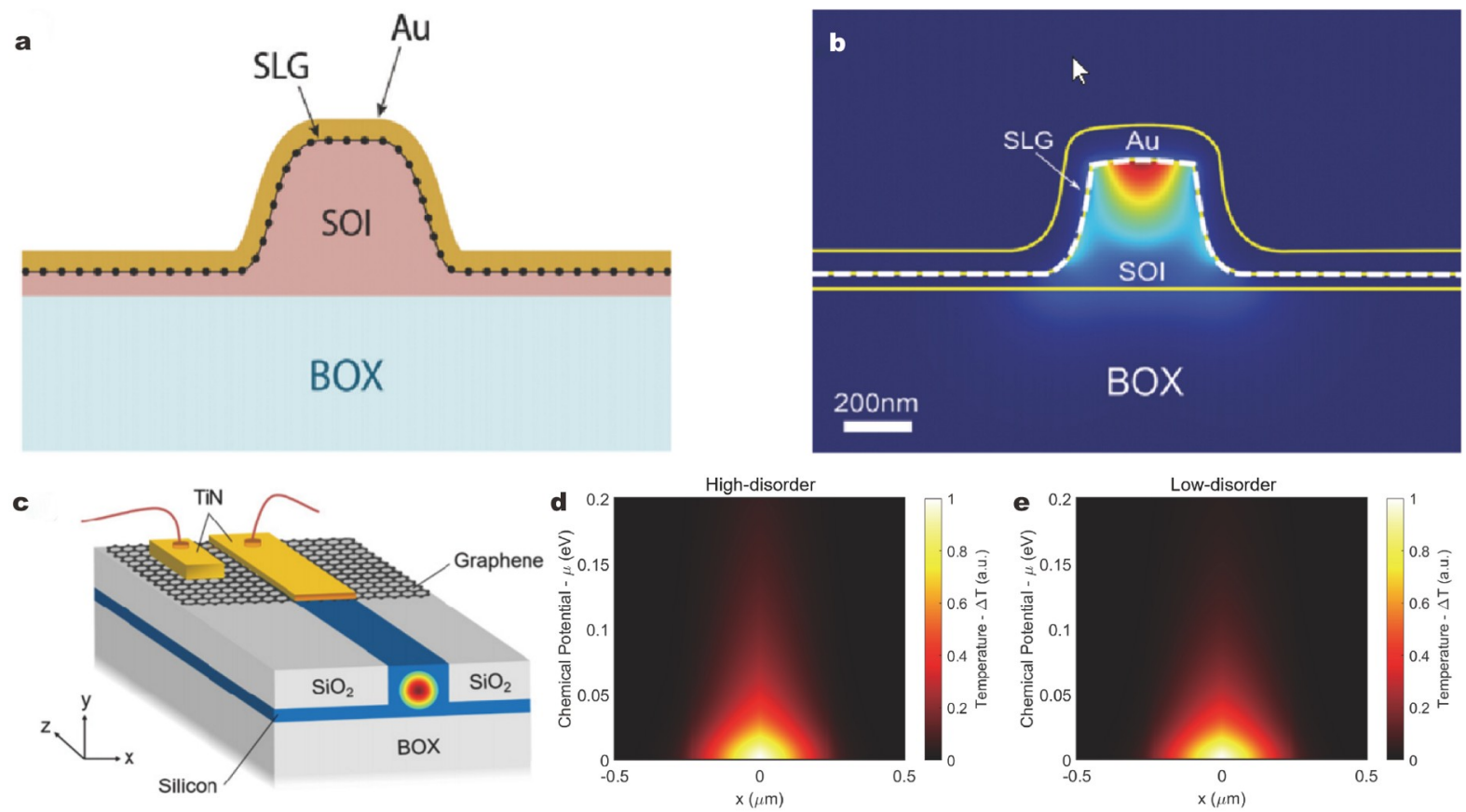

Figure 3 (a) Schematic M-SLG-Si Schottky photodetector. SOI: silicon-on-insulator. BOX: buried oxide. (b) Finite element simulated optical intensity profile of a surface plasmon polariton waveguide mode supported by an M-SLG-Si structure. (c) Schematic image of an on-chip photodetector structure. (d) Normalized temperature profiles for a varying chemical potential for (d) high-disorder (chemical potential for $\Delta=60 \mathrm{meV}$ ) and (e) low-disorder (chemical potential for $\Delta=40 \mathrm{meV}$ ) graphene sheets. (a, b) are reprinted with permission from Ref. [55]. Copyright 2016, American Chemical Society. (c-e) are reprinted with permission from Ref. [8]. Copyright 2021, Optical Society of America.

the photo-active silicon layer was reduced to $10 \mu \mathrm{m}$, suppressing the rate-limiting diffusion currents, so that significant speed improvements were obtained in both a GSOI grating device and a GSOI planar device. The rise and decay times of the former were 4 and 10 to $15 \mathrm{~ns}$, while those for the latter were 10 and 20 to $70 \mathrm{~ns}$, respectively. Recently, AlAloul and Rasras [8] used CMOS-compatible plasmonic metal $\mathrm{TiN}$ to enhance the responsivity of graphene detector on $\mathrm{Si}$ substrate (as shown in Fig. 3c). The as-mentioned TiN/graphene/Si heterostructure not only shows a high responsivity of $1.4 \mathrm{~A} \mathrm{~W}^{-1}$, but also shows a fast response speed for that its bandwidth exceeds $100 \mathrm{GHz}$. In this device, the photodetection process is predominantly determined by the PTE at zero bias, and the PTE causes a very transient Fermi-Dirac distribution of hot carriers, and they decided to use a chemical potential and a carrier temperature to describe the photodetector. The temperature profiles are shown in Fig. 3d, e.

\section{Further exploration to graphene}

On the one hand, few-layered graphene-based devices show excellent performances. Table 1 summarizes the performances of some representative devices mentioned in this paper. On the other hand, researchers have found some interesting phenomena in bilayer graphene. For bilayer graphene-based devices, a special stacking method called Bernal stacking could achieve a fieldinduced band gap which could improve the properties of graphene. In Bernal stacking bilayer graphene, half of the atoms of one layer are located exactly above the atoms of the second layer, while the other half of the atoms lie precisely above the empty center of the hexagons of the other layer. According to the theoretical calculation of Iannaccone et al. [57], the fieldinduced gap is limited to about $0.15 \mathrm{eV}$, which leads to a high on/off ratio (about 100). What's more, the twisted angle has a great influence on the properties of the bilayer graphene, Cao et al. [58-61] have published a series of papers on magic-angle twisted graphene. They fabricated a small-angle twisted bilayerbilayer graphene (TBBG) structure which consists of two sheets of Bernal-stacked bilayer graphene. Then they further studied the phases and states in the twisted bilayer graphene system [59]. The TBBG showed a rich phase diagram and tunable correlated insulator states. These insulator states exhibited high sensitivity to the twist angle and the application of electric displacement field, the latter reflecting the inherent polarizability of Bernalstacked bilayer graphene. Furthermore, this research can also provide a guide to the study of electronic correlations in other twisted van der Waals heterostructures.

Graphene is now extensively utilized for electrode materials of supercapacitors because of its high conductivity and resistance to corrosion and the graphene heterostructure-based devices have also exhibited good performances, such as broad photoresponse range, high responsivity, and ultrafast response. Different graphene-based devices have been widely used in various fields. However, we can see from the above part, these devices have many disadvantages, e.g., high-speed devices present weak photoelectric performances, while the devices with high optoelectronic properties exhibit poor response speed. More importantly, how to prepare large-scale graphene with high quality is an unavoidable problem on its road to commercial application. Improving the CVD method to prepare large-area graphene will be a development trend in the future. On the other hand, Cao's several articles [58-61], published in Science and Nature, show that graphene still has great potential to probe. Constructing nanostructure of graphene may be a research direction to explore its miraculous properties. 
Table 1 Comparison of the critical parameters for graphene-based photodetectors

\begin{tabular}{|c|c|c|c|c|c|}
\hline Devices & Wavelength (nm) & Responsivity $\left(\mathrm{A} \mathrm{W}^{-1}\right)$ & Detectivity (Jones) & Response time $(\mu \mathrm{s})$ & Ref. \\
\hline Graphene $/ \mathrm{SiO}_{2} / \mathrm{Si}$ & $514-2400$ & $6.1 \mathrm{~mA} \mathrm{~W}^{-1}$ & - & Less than $60 \mathrm{ps}$ & [47] \\
\hline Bilayer graphene $/ \mathrm{SiO}_{2} / \mathrm{Si}$ & $\mathrm{THz}$ & 4.6 & $10^{11}$ & Fast & [50] \\
\hline Nanoribbon graphene/ $\mathrm{SiO}_{2} / \mathrm{Si}$ & $\mathrm{THz}$ & 6.4 & - & Fast & [51] \\
\hline Graphene/oxide/n-Si & $410-950$ & 0.73 & $5.77 \times 10^{13}$ & $320 / 750$ & [54] \\
\hline SLG/Si Schottky & 514,1550 & 0.37 & $10^{12}$ & Fast & {$[55]$} \\
\hline Graphene/ $/ \mathrm{HfO}_{2} / \mathrm{Si}$ & $473-1150$ & 45.8 & - & $9 / 20$ & [11] \\
\hline Graphene $/ \mathrm{HfO}_{2} / \mathrm{Ge}$ & $473-1550$ & 9.03 & - & $11 / 38$ & [11] \\
\hline Graphene/ $\mathrm{HfO}_{2} / \mathrm{a}-\mathrm{MoS}_{2}$ & $473-2712$ & 5.36 & $10^{9}$ & $68 / 89$ & [11] \\
\hline TiN-graphene/Si & 1550 & 1.4 & $5 \times 10^{10}$ & Less than $10 \mathrm{ps}$ & {$[8]$} \\
\hline SLG/Ge Schottky heterojunction & - & $51.8 \mathrm{~mA} \mathrm{~W}^{-1}$ & $1.38 \times 10^{10}$ & - & {$[62]$} \\
\hline GSOI & $<1100$ & 0.26 & $7.83 \times 10^{10}$ & Several ns & {$[56]$} \\
\hline
\end{tabular}

\section{TMDC-based devices}

The common chemical formula of TMDCs is $\mathrm{MX}_{2}$, where $\mathrm{M}$ is a transition metal ( $\mathrm{M}=\mathrm{Mo}, \mathrm{W}, \mathrm{Re}$, and so on) and $\mathrm{X}$ is a chalcogen $(X=S, S e, T e)$. TMDCs possess a layered crystal structure in which the individual layers are bonded together via weak van der Waals interactions. TMDCs have many excellent properties, such as favorable flexibility and high mobility. TMDCs have a very similar structure to graphene and were considered as a substitute of graphene. Although TMDC materials have a lower mobility than graphene, they have a tunable gap and a higher light absorptivity $[63,64]$. Hence, TMDCs may be more suitable materials for optoelectronic devices compared with graphene. In fact, different TMDC materials have different characteristics. In this section, hence, we will introduce basic properties of some TMDC materials, followed by versatile applications. And as a most typical TMDC material, $\mathrm{MoS}_{2}$ will be the focus of a large section.

\section{$\mathrm{MoS}_{2}$-based devices}

As a typical low-dimensional layered TMDCs, $\mathrm{MoS}_{2}$ has been extensively studied due to its exciting properties. $\mathrm{MoS}_{2}$ is a typical isotropic crystal whose bandgap is thickness-dependent. The bandgap of bulk $\mathrm{MoS}_{2}$ is $1.2 \mathrm{eV}$ which is an indirect gap, while single-layer $\mathrm{MoS}_{2}$ is a direct-gap material with a bandgap of $1.8 \mathrm{eV}$ [65]; this phenomenon attract scientists' interest and they focus more attention on few-layer $\mathrm{MoS}_{2}$-based devices. For example, Wang et al. [24] reported the construction of few-layer $\mathrm{MoS}_{2} / \mathrm{Si}$ heterojunction-based photodetectors. The $\mathrm{MoS}_{2} / \mathrm{Si}$ device showed a wide photoresponse range of $450-1050 \mathrm{~nm}$, ultrafast response speed of $3 \mu$ s, and high detectivity of $10^{13}$ Jones. Although $\mathrm{MoS}_{2}$-based photodetectors have excellent performances, the intrinsic bandgap of $\mathrm{MoS}_{2}$ (whose cutoff wavelength edge is about $1 \mu \mathrm{m}$ ) limits the detection range of the photodetectors. The deviation from the perfection of the atomic lattice, which can effectively tune the bandgap and response range, may provide an appropriate way to broaden the detection range of the $\mathrm{MoS}_{2}$ photodetectors. In 2016, Xie et al. [66] fabricated an ultrabroadband multilayer $\mathrm{MoS}_{2} / \mathrm{Si}$ photodetector by controlling the defects of S atoms. They designed $\mathrm{MoS}_{2}$ with an element ratio of S/Mo ions of 1.89, and found the bandgap was decreased to $0.26 \mathrm{eV}$ in a theoretical calculation, and the cutoff wavelength could reach about $4 \mu \mathrm{m}$. The as-fabricated photodetectors showed a wide response region up to $4.7 \mu \mathrm{m}$, and were investigated in the wavelength range from 445 (blue light) to 2717 (mid-infrared (MIR)) nm. At a cost, the responsivity and detectivity dropped to $50.7 \mathrm{~mA} \mathrm{~W}^{-1}$ and $1.55 \times 10^{9}$ Jones, respectively. However, due to the lack of high-quality $p-n$ junction and large wafer scale fabrication process, the $\mathrm{MoS}_{2}$ based photodetectors are limited for commercial applications. Recently, large-scale preparations for TMDC/Si heterostructurebased devices have been realized. Dhyani et al. [20] successfully fabricated a large-area $\mathrm{MoS}_{2} / \mathrm{Si}$ heterostructure-based photodetector with a simple, reproducible and scalable fabrication process. This photodetector was compatible to CMOS circuits and had excellent performances, such as high responsivity (up to $8.75 \mathrm{~A} \mathrm{~W}^{-1}$ ), high detectivity (about $1.4 \times 10^{12}$ Jones) and fast response time (about $10 \mu \mathrm{s}$ ), and the transient measurements of the phototransistors showed excellent stable and reproducible dynamic response to high-frequency laser.

On the other hand, Wang et al. [67] successfully demonstrated an ultrasensitive and broadband photodetector in another way. In this device, they made pioneering use of poly(vinylidene fluoride-trifluoroethylene) ( $\mathrm{P}(\mathrm{VDF}-\mathrm{TrFE}))$, an organic ferroelectric material, and the remnant polarization of $\mathrm{P}(\mathrm{VDF}-\mathrm{TrFE})$ provided a high local electrostatic field, making the few-layer $\mathrm{MoS}_{2}$ channel a fully depleted state which significantly increased the sensitivity of the detector. Possessing these novel properties, the photodetector attained a responsivity of up to $2570 \mathrm{~A} \mathrm{~W}^{-1}$, along with the detectivity of $2.2 \times 10^{12}$ Jones, as well as a broad response range of $450-1550 \mathrm{~nm}$. It is worth noting that the $\mathrm{MoS}_{2} / \mathrm{P}(\mathrm{VDF}-\mathrm{TrFE})$ heterostructure exhibited pronounced photovoltaic activity, enabling their applications as self-driven photodetectors operated at zero bias voltage. It means that the low energy-cost photodetectors can be considered for applications in portable and flexible devices. This successful sample provides a new way, utilizing hybrid heterostructures and nanocomposites, to boost the performances of $\mathrm{MoS}_{2}$ photodetectors. In fact, diverse $\mathrm{MoS}_{2}$ hybrid heterostructures could increase carrier mobility and incident light absorption. For example, Xiao et al. [68] reported a 3D reduced graphene oxide (RGO)- $\mathrm{MoS}_{2}$ /pyramid $\mathrm{Si}$ heterostructure-based photodetector which was made by a simple solution-processing method. Fig. 4 shows a schematic illustration of the structure of the $3 \mathrm{D}$ heterostructure-based photodetector, current-voltage characteristics of the device, a comparison of absorption spectra of planar and pyramid $\mathrm{Si}, \mathrm{RGO}$, and $\mathrm{RGO}-\mathrm{MoS}_{2} / \mathrm{Si}$ heterojunction devices and 
a

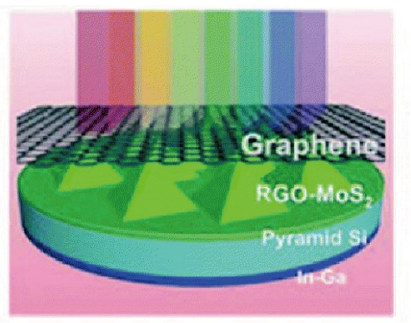

d

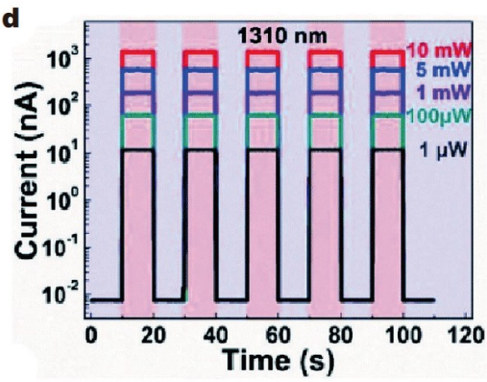

b

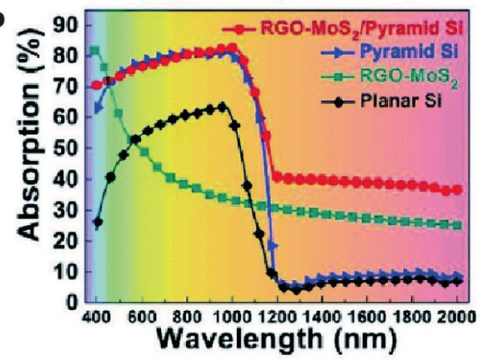

e

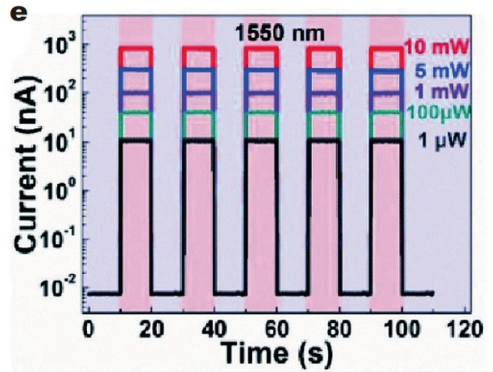

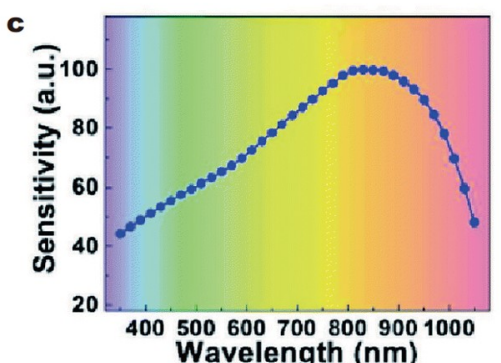

Wavelength $(\mathrm{nm})$

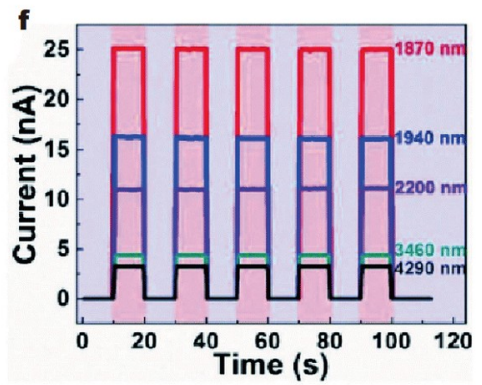

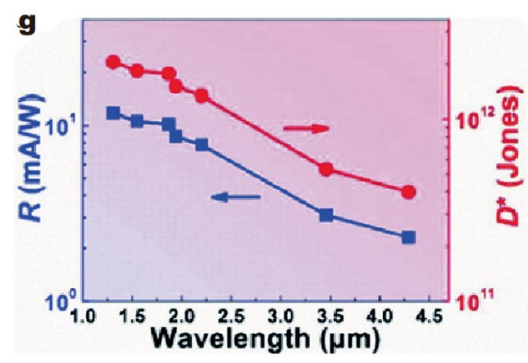
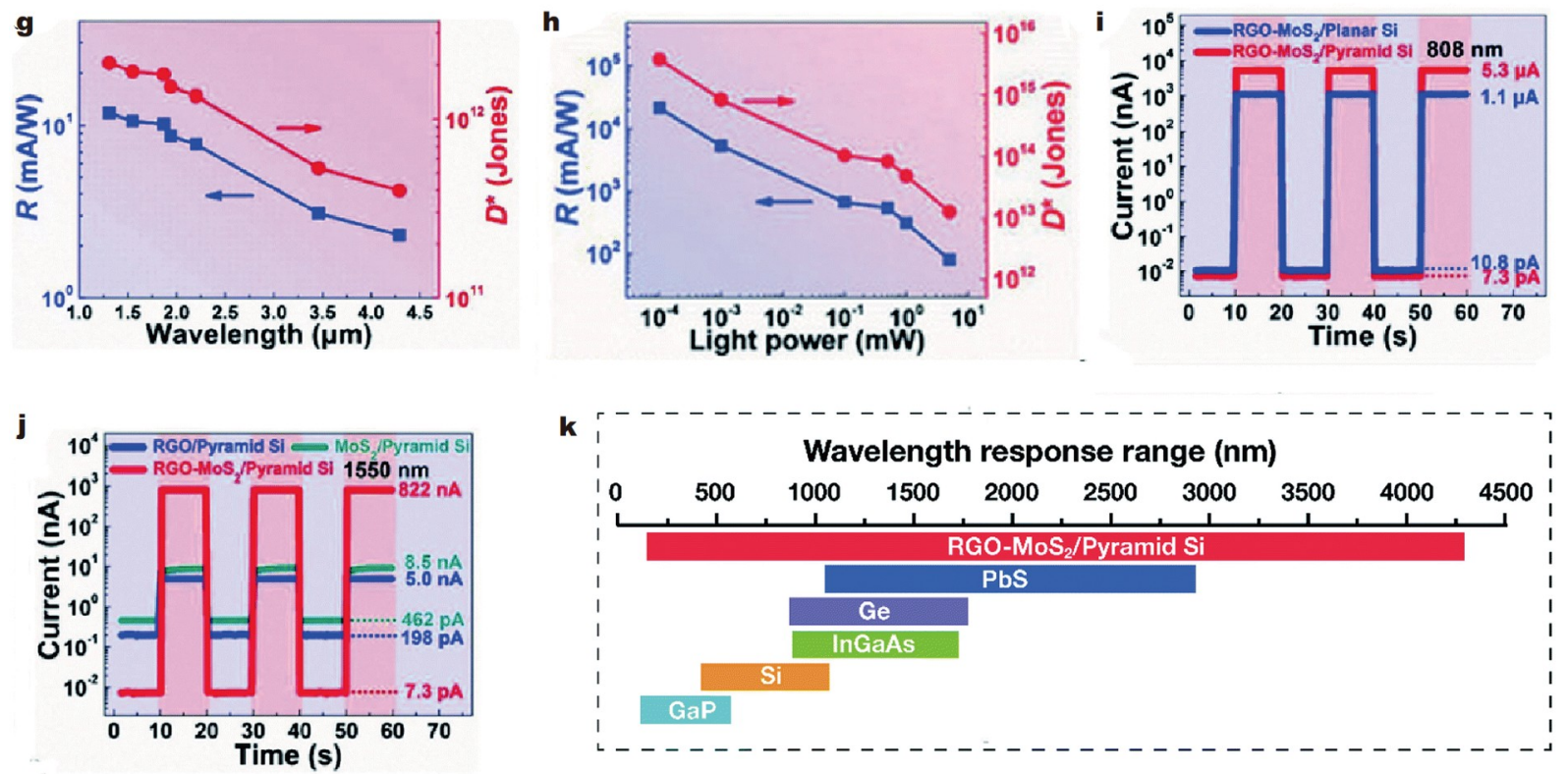

Figure 4 (a) Schematic illustration of the RGO- $\mathrm{MoS}_{2} /$ pyramid Si heterojunction-based photodetector. (b) A comparison of the absorption spectra of planar and pyramid Si, RGO, and RGO-MoS $/$ Si heterojunction devices. (c) Photosensitivity of the RGO-MoS $/$ /pyramid Si heterojunction-based device in the wavelength region between 350 and $1100 \mathrm{~nm}$. (d, e) Photocurrent switching behavior of the heterojunction device measured under light illuminations at (d) 1310 and (e) $1550 \mathrm{~nm}$ at zero bias voltage. (f) Photocurrent switching behavior of the heterojunction device measured under 1870, 1940, 2200, 3460 and $4290 \mathrm{~nm}$ (near-infrared (NIR)-MIR) light illuminations. (g) Photoresponsivity and specific detectivity curves of the heterojunction device measured as a function of wavelength from 1310 to $4290 \mathrm{~nm}$ at a fixed laser power intensity of $50 \mathrm{~mW}$ under zero bias voltage. (h) $R$ and $D^{*}$ curves of the heterojunction photodetector measured as a function of laser power intensity at $808 \mathrm{~nm}$ under zero bias voltage. (i, j) Comparison of the photocurrent switching behavior of RGO- $-\mathrm{MoS}_{2} /$ pyramid Si and RGO- $\mathrm{MoS}_{2} /$ planar Si heterojunction devices measured at (i) $808 \mathrm{~nm}, 1 \mathrm{~mW}$ laser power intensity, and (j) $1550 \mathrm{~nm}, 10 \mathrm{~mW}$ laser power intensity. (k) A comparison of the wavelength coverage by the RGO- $\mathrm{MoS}_{2} /$ pyramid Si heterojunction-based photodetector along with other traditional semiconductor-based photodetectors. The RGO- $\mathrm{MoS}_{2} /$ pyramid Si hybrid heterojunction-based photodetectors operated from 350 to $4.3 \mathrm{~mm}$ (UV to MIR) ultra-broadband spectral range due to the bandgap narrowing caused by the $\mathrm{S}$ vacancy defects in $\mathrm{MoS}_{2}$ crystals. (a-j) Reprinted with permission from Ref. [68]. Copyright 2018, Wiley-VCH Verlag GmbH \& Co. KGaA, Weinheim. (k) Reprinted with permission from Ref. [69]. Copyright 2020, The Royal Society of Chemistry.

diverse performances of the 3D heterostructure photodetector. From the figure, we can see, at zero bias voltage, the photovoltage of RGO- $\mathrm{MoS}_{2} / \mathrm{Si}$ heterostructure photodetector increases with the enhancement of incident light power intensity, indi- cating the self-powered operation of the photodetector. Furthermore, this $3 \mathrm{D}$ heterostructure-based photodetector exhibited excellent performances because of the special structure, such as an extreme broad spectrum range from ultraviolet (UV) 
$(350 \mathrm{~nm})$ to MIR (4300 nm); moreover, a very high detectivity of $3.8 \times 10^{15}$ Jones and a photoresponsivity of $21.8 \mathrm{~A} \mathrm{~W}^{-1}$ were achieved at the illumination of $808-\mathrm{nm}$ incident light.

With the continuous study of $\mathrm{MoS}_{2}$, researchers found $\mathrm{MoS}_{2}$ possesses three phases: $2 \mathrm{H}-\mathrm{MoS}_{2}, 3 \mathrm{R}-\mathrm{MoS}_{2}$ and $1 \mathrm{~T}-\mathrm{MoS}_{2}$, where $\mathrm{H}, \mathrm{R}$, and $\mathrm{T}$ represent hexagonal, rhombohedral and tetragonal symmetry, respectively, and " 1 ", “ 2 ", and " 3 " represent the number of S single bonds, Mo single bonds, and S layers within a single unit cell, respectively [70]. Different phases have different structures and properties. Fig. 5a-f show crystal structures and Raman spectra of the three phases and the other $\mathrm{MoX}_{2}$ have similar structures. Common semiconducting $\mathrm{MoS}_{2}$ in nature is $2 \mathrm{H}$ phase, while the metallic $1 \mathrm{~T}-\mathrm{MoS}_{2}$ is metastable and can be synthesized by utilizing the interaction of alkali metal ions. What we have discussed in the previous part is also $2 \mathrm{H} \mathrm{MoS} 2$ [71]. As we can see from the previous part, the $2 \mathrm{H}-\mathrm{MoS}_{2}$-based photodetectors have the disadvantage of low photoresponse which severely limits their broader applications in optoelectronic fields. The metallic $1 \mathrm{~T}-\mathrm{MoS}_{2}$ [72] shows ultrafast response time which complements $2 \mathrm{H}-\mathrm{MoS}_{2}$ very well. Recently, Wang et al. [73] fabricated vertically stacked $2 \mathrm{H}-\mathrm{MoS}_{2} / 1 \mathrm{~T} @ 2 \mathrm{H}-\mathrm{MoS}_{2} / \mathrm{Si}$ photodetectors which integrated the positive traits of $2 \mathrm{H}-\mathrm{MoS}_{2}$ and $1 \mathrm{~T}-\mathrm{MoS}_{2}$. The hybrid $\mathrm{MoS}_{2} / \mathrm{Si}$ heterostructure showed fewer dangling bonds and better lattice matching which ensured an excellent performance with responsivity of $1917 \mathrm{~A} \mathrm{~W}^{-1}$, detectivity of $7.55 \times 10^{11}$ Jones, and EQE of $448,384 \%$ at $532 \mathrm{~nm}$ wavelength light illumination with gate bias of $20 \mathrm{~V}$. The very high EQE value is caused by Schottky barrier modulation. As a comparison, the maximum $R$ of $1 \mathrm{~T} @ 2 \mathrm{H}-\mathrm{MoS}_{2}$-based photodetector is about $10^{-4} \mathrm{~A} \mathrm{~W}^{-1}$ which is even lower than that of the $2 \mathrm{H}-\mathrm{MoS}_{2}$-based transistor (maximum value of $56 \mathrm{~A} \mathrm{~W}^{-1}$ ), indicating that the $1 \mathrm{~T} @ 2 \mathrm{H}-\mathrm{MoS}_{2}$ layer only provides high-speed carrier and the effect of the $1 \mathrm{~T} @ 2 \mathrm{H}-\mathrm{MoS}_{2}$ layer is negligible in the photodetection performance. The schematic diagram is show a

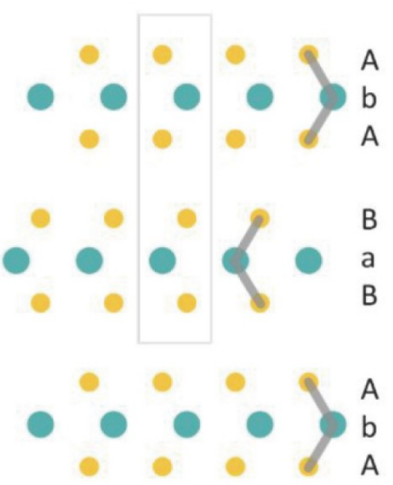

b

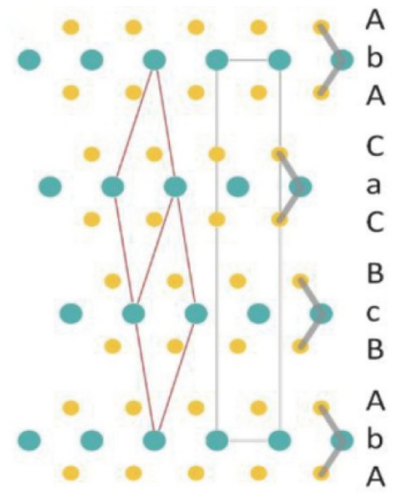

c

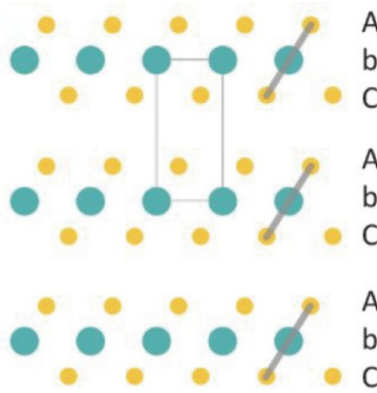

S Mo

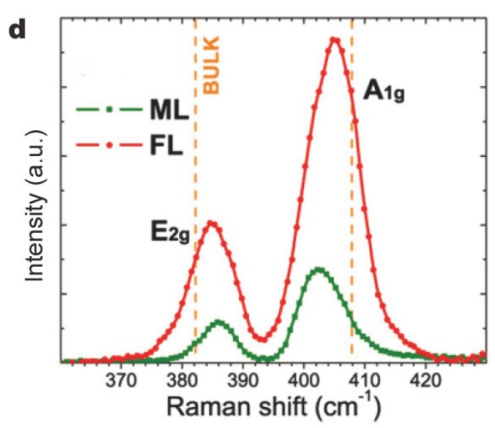

g

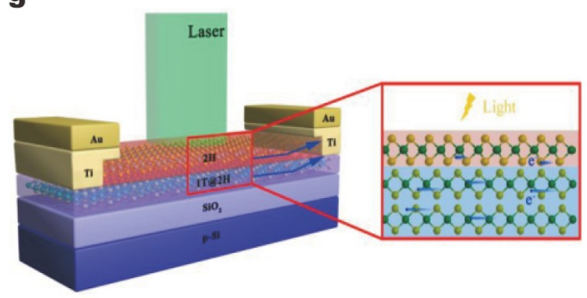

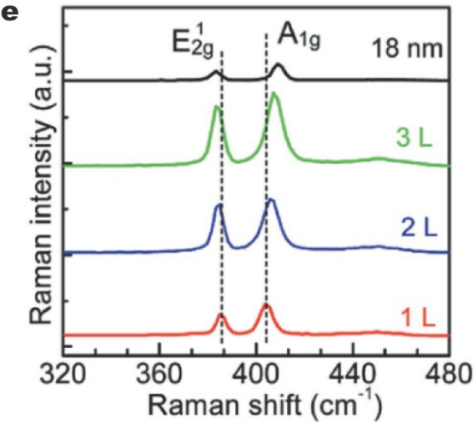

h

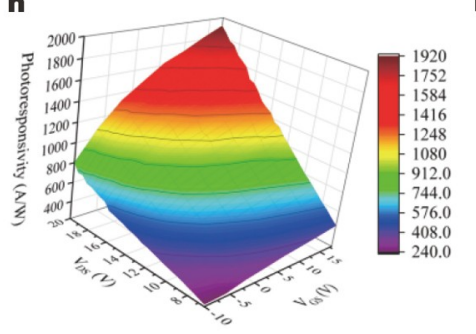

f

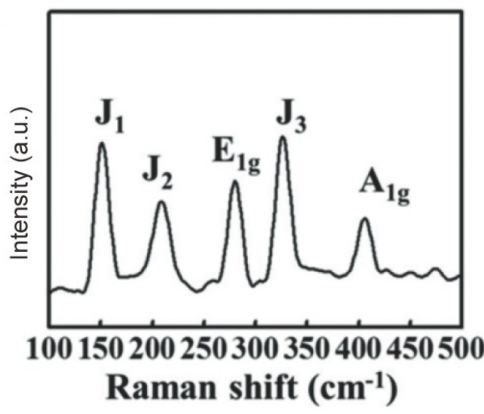

i

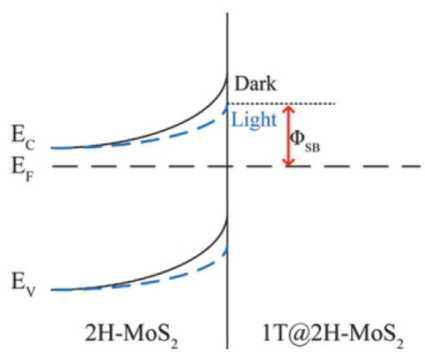

Figure 5 (a) $2 \mathrm{H}$, (b) $3 \mathrm{R}$, and (c) $1 \mathrm{~T}$ phases of $\mathrm{MoS}_{2}$. Crystals viewed along the $a$-axis, where the unit cell is shown as a gray box (3R rhombohedral unit cell shown in red). (a-c) are reprinted from Ref. [74]. Copyright 2021, American Chemical Society. (d) Raman spectrum of 2H MoS 2 ML: monolayer; FL: fewlayer. Reprinted from Ref. [75]. Copyright 2021, American Chemical Society. (e) Raman spectrum of 3R $\mathrm{MoS}_{2}$. Reprinted from Ref. [76]. Copyright 2017, WILEY-VCH Verlag GmbH \& Co. KGaA, Weinheim. (f) Raman spectrum of 1T MoS 2 . Reprinted from Ref. [77]. Copyright 2018, Wiley-VCH Verlag GmbH \& Co. KGaA, Weinheim. (g) 3D graphic demonstration showing the typical photodetector structure on the left. The zoomed-in region in the red box on the right presents transported electrons moving in different layers. (h) 3D view of photoresponsivity mapping for this photodetector under different gate bias and source-drain bias values, at an illumination power density of $2.35 \mathrm{~mW} \mathrm{~cm}{ }^{-2}$. (i) Schematic band diagram of the semiconducting $2 \mathrm{H}-\mathrm{MoS}_{2}$ and $\mathrm{metal}-\mathrm{like}$ 1T@2H-MoS 2 diodes. (g-i) are reprinted from Ref. [73]. Copyright 2020, American Chemical Society. 
in Fig. 5g.

\section{Other Mo- and W-dichalcogenide-based devices}

Unlike $\mathrm{MoS}_{2}, \mathrm{MoSe}_{2}$ is a semiconducting material with an indirect bandgap of $1.1 \mathrm{eV}$ in bulk form, which increases gradually to $1.55 \mathrm{eV}$ and becomes direct bandgap in monolayer and bilayer. The monolayer $\mathrm{MoSe}_{2}$-based FET shows an n-type behavior and its average mobility is about $50 \mathrm{~cm}^{2} \mathrm{~V}^{-1} \mathrm{~s}^{-1}$, while the mobility of bulk $\mathrm{MoSe}_{2}$ is about $100 \mathrm{~cm}^{2} \mathrm{~V}^{-1} \mathrm{~s}^{-1}[78,79]$. In 2019, a $\mathrm{MoSe}_{2} / \mathrm{p}$-Si junction was successfully demonstrated for position-sensitive detector [80]. The $\mathrm{MoSe}_{2} / \mathrm{Si}$ showed a lateral PVE (LPE) which exhibited a good liner dependence on the laser spot position. When there was a small displacement of the laser spot, the LPE caused a relative change in the corresponding current. As a consequence, the $\mathrm{MoSe}_{2} / \mathrm{p}$-Si junction showed a large positional sensitivity of $563 \mathrm{mV} \mathrm{mm}^{-1}$. Moreover, $\mathrm{MoSe}_{2}$ has a higher optical absorption and a narrower bandgap; hence, the $\mathrm{MoSe}_{2} / \mathrm{Si}$ is also a good alternative used for infrared detection. Jie's group [81] fabricated graphene/ $\mathrm{MoSe}_{2} / \mathrm{Si}$ photodetectors. In this heterostructure, the graphene layer was used for transparent electrode to collect carriers. Fig. $6 a, d$ and e show the structure of the detector and the carrier collecting effect of graphene transparent electrode. The as-mentioned photodetector was sensitive to the light from 365 to $1310 \mathrm{~nm}$ with ultrafast response (about $0.3 \mu$ s for both raise and fall time). Meanwhile, the detectivity and responsivity of the photodetector were $7.13 \times 10^{10}$ Jones and $0.27 \mathrm{~A} \mathrm{~W}^{-1}$, respectively. Ko et al. [82] introduced a $\mathrm{SiO}_{2}$ layer to few-layered $\mathrm{MoSe}_{2} / \mathrm{Si}$ heterostructure to form a back-gated phototransistor. The photoresponsivity could be easily controlled to reach a maximum value of $238 \mathrm{~A} \mathrm{~W}^{-1}$ under NIR light excitation, while achieving a high specific detectivity $D^{*}=7.6 \times 10^{11}$ Jones, a high EQE of $37,745 \%$. In a recent report, Xu et al. [40] synthesized a stacked layered $\mathrm{MoSe}_{2}$ on an n-type Si substrate. The layered $\mathrm{MoSe}_{2}$ was uniform and with high quality and the device exhibited excellent performances. The $\mathrm{n}-\mathrm{n} \mathrm{MoSe}_{2} / \mathrm{Si}$ heterojunction showed a broad response spectrum up to $1500 \mathrm{~nm}$. The responsivity and detectivity can reach up to $0.58 \mathrm{~A} \mathrm{~W}^{-1}$ and $1.4 \times 10^{14}$ Jones, respectively. These performances are much better than those of most previous $\mathrm{MoSe}_{2}$-based photodetectors which are mainly deter- a

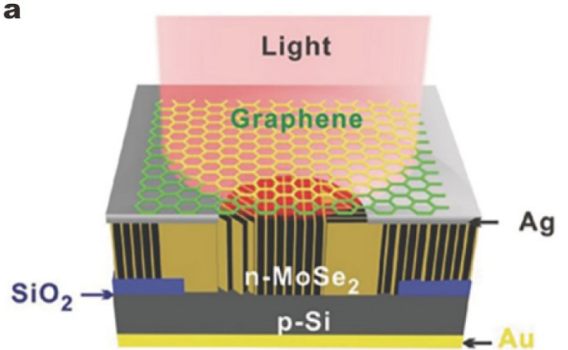

b

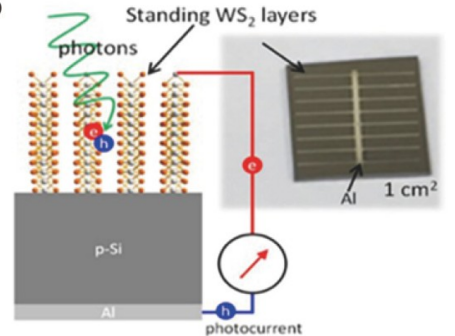

d

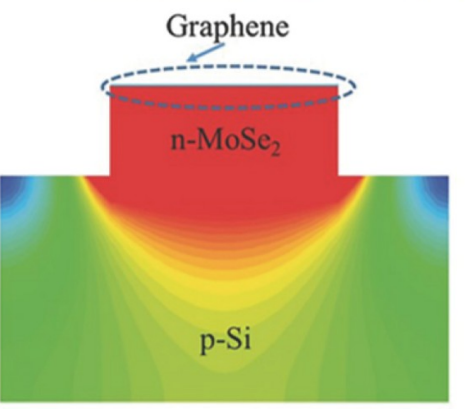

$\mathbf{g}$

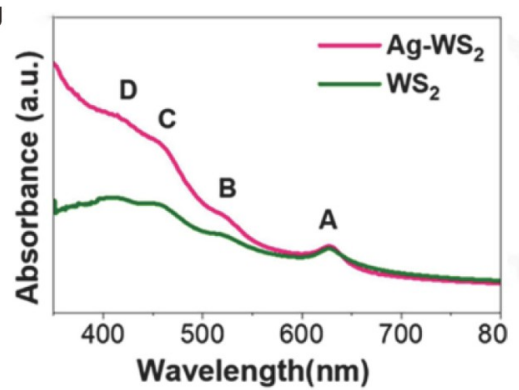

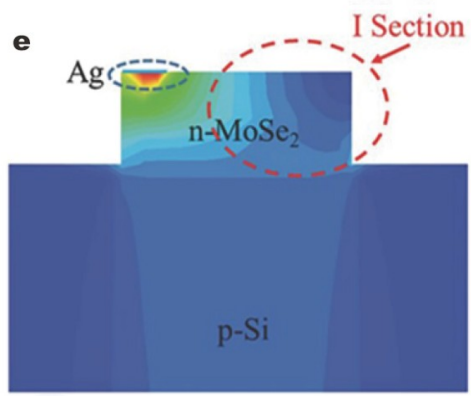

h

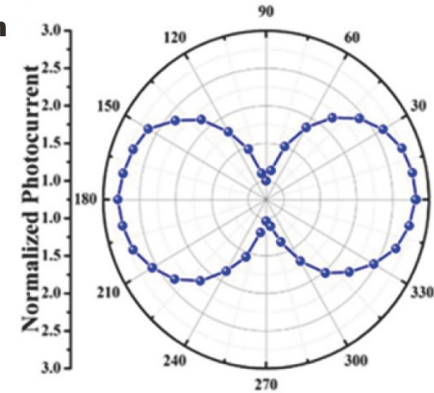

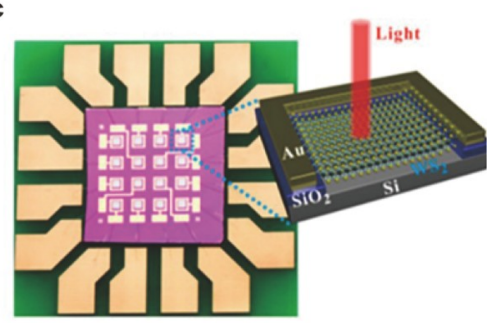

f Total Current Density
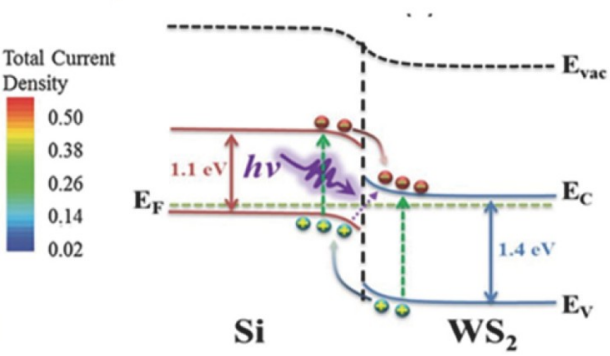

i

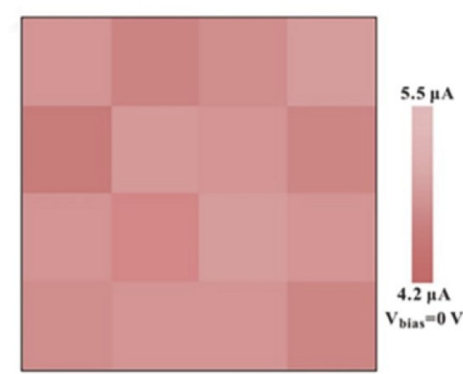

Figure 6 (a) Schematic illustration of the graphene/ $\mathrm{MoSe}_{2} / \mathrm{Si}$ heterojunction photodetector with graphene transparent electrode. (b) Schematic and photograph of the $\mathrm{WS}_{2} / \mathrm{p}$-Si heterojunction device. (c) The photograph of $\mathrm{WS}_{2} / \mathrm{Si}$ array device. Simulated total current density distributions of the $\mathrm{MoSe}_{2} / \mathrm{Si}$ photodetectors (d) with and (e) without graphene top electrode at forward bias of $+1 \mathrm{~V}$, respectively. (f) The band diagram of $\mathrm{WS}_{2} / \mathrm{Si}_{\mathrm{s}}$ heterostructure. (g) Absorption spectra of Ag-WS 2 nanocomposite compared with $\mathrm{WS}_{2}$. (h) Normalized photocurrent of the $\mathrm{WS}_{2} / \mathrm{Si}$ heterojunction as a function of the polarization angle. (i) Spatial resolved photocurrent mapping of a $4 \times 4$ heterojunction device array. (a, d, e) are reprinted with permission from Ref. [81]. Copyright 2016, The Authors. Published by WILEY-VCH Verlag GmbH \& Co. KGaA, Weinheim. (b) is reprinted with permission from Ref. [84]. Copyright 2018, American Chemical Society. (c, f, h and i) are reprinted with permission from Ref. [85]. Copyright 2019, American Chemical Society. (g) is reprinted with permission from Ref. [86]. Copyright 2020, Elsevier B.V. 
mined by the high-quality $\mathrm{MoSe}_{2}$ film. In addition, the response speed approaches $0.12 \mu \mathrm{s}$, meaning the device may be used in high-speed circuits. Almost the same time, John et al. [83] fabricated a $\mathrm{MoSe}_{2} / \mathrm{Si}$ p-n junction photodiode whose spectral range covered 405 to $2500 \mathrm{~nm}$, and the maximum responsivity of $522 \mathrm{~mA} \mathrm{~W}^{-1}$ was attained at the illumination of $1100 \mathrm{~nm}$ incident light. The $\mathrm{MoSe}_{2}$ nanosheets were synthesized by a hydrothermal method, causing many defects in the lattice which was the main reason for high optical absorption of the shortwave infrared light. Furthermore, the response speed of the devices was also fast, for that the rise time was $18 \mu \mathrm{s}$ and the fall time was $14 \mu$ s in the infrared region.

$\mathrm{MoTe}_{2}$ has an indirect bandgap of $1.0 \mathrm{eV}$ and a high carrier mobility of $200 \mathrm{~cm} \mathrm{~V}^{-1} \mathrm{~s}^{-1}$ in its bulk form, which also has strong light absorption so that it is usually used for electrode materials in photovoltaic cells; while in its monolayer form, $\mathrm{MoTe}_{2}$ has a direct bandgap of $1.07 \mathrm{eV}$. In 2016, Octon et al. [87] observed a fast photoresponse time $(\sim 160 \mu \mathrm{s})$ in few-layered $\mathrm{MoTe}_{2}$-based photodetectors with a high responsivity of $6 \mathrm{~A} \mathrm{~W}^{-1}$. However, this $\mathrm{MoTe}_{2}$-based photodetector has a narrow spectral range. Recently, Lu et al. [88] fabricated a few-layer $\mathrm{MoTe}_{2} / \mathrm{Si}$ vertical heterojuction which has broad response band (from 300 to $1800 \mathrm{~nm}$ ) and a very fast response speed. The ntype $\mathrm{MoTe}_{2}$ nanosheet was synthesized on an n-type Si substrate via a pulsed laser deposition (PLD) method, and graphene was selected as the transparent electrode to ensure an ohmic contact with $\mathrm{MoTe}_{2}$ and a high optical absorption. The n-n heterostructure photodetector exhibited a responsivity of $0.19 \mathrm{~A} \mathrm{~W}^{-1}$, a large detectivity of $6.8 \times 10^{13}$ Jones and a high current on/off ratio of $3.2 \times 10^{6}$ at $980 \mathrm{~nm}$. It is noted that the device possesses an ultrahigh speed of a rise time of $150 \mathrm{~ns}$ and a fall time of $350 \mathrm{~ns}$. They used laser with different pulse frequencies $(100 \mathrm{~Hz}$ $-5 \mathrm{MHz}$ ) to illuminate the device and recorded the signal. The 3$\mathrm{dB}$ optical bandwidth was estimated to be up to $1.0 \mathrm{MHz}$ and the electrical bandwidth was calculated to be $0.12 \mathrm{GHz}$. The ideality factor $(n)$ of the $\mathrm{MoTe}_{2} / \mathrm{Si}$ heterojunction was 1.9 , in contrast to the much larger value of 3.5 for graphene/Si Schottky junction. In addition, the turn-on voltage of the device was $0.15 \mathrm{~V}$ and the saturation current was $20 \mu \mathrm{A}$ at a reverse voltage of $-10 \mathrm{~V}$. Just like $\mathrm{MoS}_{2}, \mathrm{MoTe}_{2}$ also has two common phases: $1 \mathrm{~T}$ phase and $2 \mathrm{H}$ phase, while $1 \mathrm{~T}-\mathrm{MoTe}_{2}$ is metallic and metastable. In a latest study, Lin et al. [89] inserted $1 \mathrm{~T}-\mathrm{MoTe}_{2}$ into $2 \mathrm{H}-\mathrm{MoTe}_{2}$ to improve the photoresponse properties of $\mathrm{MoTe}_{2}$-based photodetector. The 1T-MoTe $e_{2}$ was introduced to enhance the interlayer reaction of the $\mathrm{MoTe}_{2}$ nanosheet. The responsivity was enhanced about 10 times compared with single $2 \mathrm{H}-\mathrm{MoTe}_{2}$-based photodetector, and the response time decreased from about 190 to about $90 \mu \mathrm{s}$.

$\mathrm{WX}_{2}$ materials have similar properties to $\mathrm{MoX}_{2}$, and many researchers study these materials from different perspectives to dig inner details of them. For example, Zheng et al. [90] tried to combine few-layered $\mathrm{WSe}_{2}$ with different substrates (including $\mathrm{Si} / \mathrm{SiO}_{2}$, polyimide (PI) and glass) to develop different heterostructures. The $\mathrm{WSe}_{2}$ thin films were prepared on various substrates by using PLD and indium tin oxide (ITO) was deposited as transparent electrodes. And the response range of the asfabricated devices was $370-1064 \mathrm{~nm}$, while the responsivity could reach up to $0.92 \mathrm{~A} \mathrm{~W}^{-1}$, along with the maximum EQE of $180 \%$. Also, Pataniya's group [91] designed a $\mathrm{WSe}_{2} / \mathrm{Si}$ hetero junction, with an ideality factor of 1.21 and a faster response time of $32 \mathrm{~ms}$ (compared with Zheng's $0.9 \mathrm{~s}$ ).
The $\mathrm{WS}_{2} / \mathrm{Si}$ heterojunction also exhibited excellent performances. In 2018, Kim et al. [84] fabricated a $\mathrm{WS}_{2} / \mathrm{p}$-Si vertical heterostructure. This photodetector showed a broad response range which covers form UV to NIR (360-850 nm) and an ultrafast photoresponse $(1.1 \mu \mathrm{s})$ along with a specific detectivity of $1.5 \times 10^{10}$ Jones; however, the responsivity is low $\left(4 \mathrm{~mA} \mathrm{~W}^{-1}\right)$. It is worth noting that the area of $\mathrm{WS}_{2}$ is very large to wafer scale along with ultrahigh response speed, indicating a potential application in the integrated circuits design. In a latest report, $\mathrm{Ag}$ nanoparticles were decorated on $\mathrm{WS}_{2}$ nanosheets via an LPE to form a metal/TMDCs nanocomposite [86]. Just like the TiN/ graphene heterostructure, the $\mathrm{Ag} / \mathrm{WS}_{2}$ structure would provide an improved plasmonic effect which can enhance the light absorption and photodetection ability of the device. Fig. $6 \mathrm{~g}$ shows the enhanced light absorption of the nanocomposite. The as-made $\mathrm{Ag}-\mathrm{WS}_{2} / \mathrm{Si}$ exhibited a higher responsivity of $2.09 \mathrm{~A} \mathrm{~W}^{-1}$, a specific detectivity of $6.6 \times 10^{11}$ Jones, and a high EQE of $487 \%$. In a recent study, Wu et al. [85] extended the detection range of $\mathrm{WS}_{2} / \mathrm{Si}$ heterojunction detector to MIR (Fig. 6c). They explained the interlayer excitation was generated by the small gap between valence band maximum of $\mathrm{Si}$ and conduction band minimum of $\mathrm{WS}_{2}$ (Fig. 6f). Therefore, the detectors could be sensitive to MIR light with a wavelength below the bandgaps of $\mathrm{WS}_{2}$ and $\mathrm{Si}$ for that the detector would be sensitive to the $3043 \mathrm{~nm}$ infrared light. In addition, they made a $4 \times 4$ array to enhance the optoelectronic performance of the detector (Fig. 6i). Moreover, the photodetector exhibit a high $I_{\text {on }} / I_{\text {off }}$ ratio of $>10^{6}$. In addition, the $\mathrm{WS}_{2} / \mathrm{Si}$ heterojunction shows sensitivity to polarized light and a high peak-to-valley ratio of 2.8 (Fig. $6 \mathrm{~h}$ ).

\section{Group-ten dichalcogenide-based devices}

In recent years, more and more studies of group-ten TMDCs are emerging due to their unique structures and electrical properties [92-94]. For group-ten TMDCs/Si heterostructures, $\mathrm{MSe}_{2}(\mathrm{M}=$ $\mathrm{Pd}, \mathrm{Pt}$ ) is more commonly used to construct the hybrid structures. For example, researchers directly grow $\mathrm{PdSe}_{2}$ film on $\mathrm{Si}$ and pyramid Si substrates to form heterojunctions, respectively $[95,96]$ (see Fig. 7a, b). These two heterojunctions exhibit similar performances and some parameters may be enhanced by the structure of pyramid Si. Fig. 7c shows the electric field energy distribution of the $\mathrm{PdSe}_{2} /$ pyramid-Si. The responsivity of $\mathrm{PdSe}_{2} /$ pyramid-Si is about $450 \mathrm{~mA} \mathrm{~W}^{-1}$ compared with the $\mathrm{PdSe}_{2} / \mathrm{Si}^{\prime} \mathrm{s}$ $300 \mathrm{~mA} \mathrm{~W}^{-1}$, and the detectivity of $\mathrm{PdSe}_{2} /$ pyramid-Si is also a little higher. The on/off ratios of them are both at a $10^{5}$ magnitude. It is noted that the $\mathrm{PdSe} / \mathrm{Si}$ heterostructure detector has an ultrabroad response range covering form deep ultraviolet (DUV) $(200 \mathrm{~nm})$ to MIR (3044 nm), while the PdSe $2 /$ pyramid-Si has a successful trail on infrared imaging (see Fig. 7d-f). Aftab et al. [97] combined $\mathrm{n}-\mathrm{PdSe}_{2}$ with p-type $\mathrm{Si}$ substrate by an $\mathrm{ME}$ technique. For fine electrodes, e-beam lithography was performed to produce $\mathrm{Al}_{2} \mathrm{O}_{3}$ ( $25 \mathrm{~nm}$ thick) films. The as-fabricated photodetector showed higher responsivity of $47.2 \mathrm{~A} \mathrm{~W}^{-1}$ and lower detectivity of $8.13 \times 10^{9}$. These two methods to make heterostructures cause different results, which may be due to the inconsistent crystal quality and the degree of bonding between the $\mathrm{PdSe}_{2}$ films and the substrates.

$\mathrm{PtSe}_{2}$ has a prominent layer-dependent electronic behavior, i.e., the monolayer $\mathrm{PtSe}_{2}$ is semiconducting while the bulk form is semi-metallic. In a latest report, the wafer-scale [98] $\mathrm{PtSe}_{2}$ film was directly grown on Si substrate to form a photocathode via a 
a

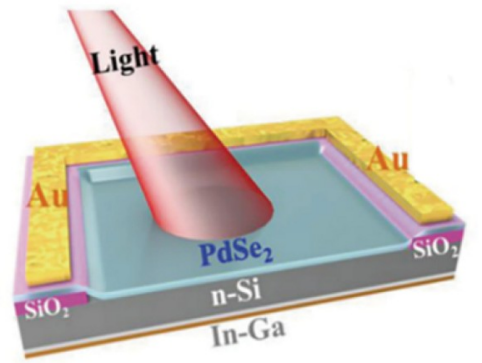

d

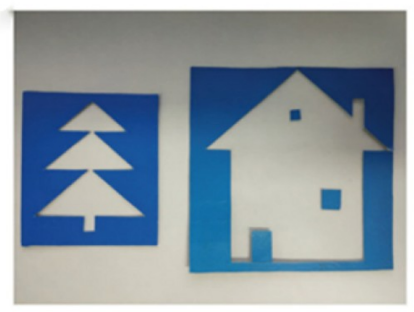

b

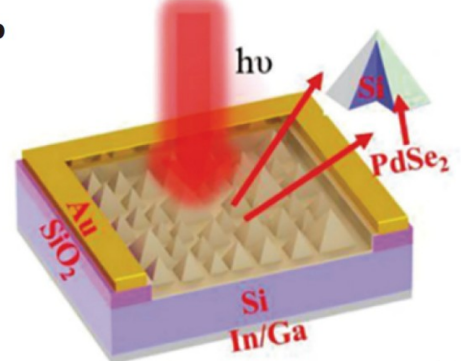

e

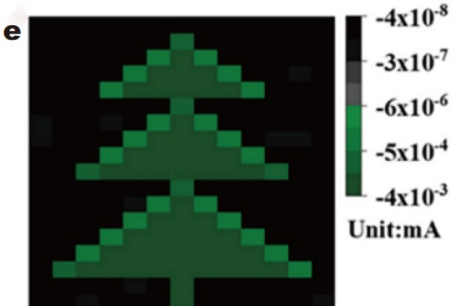

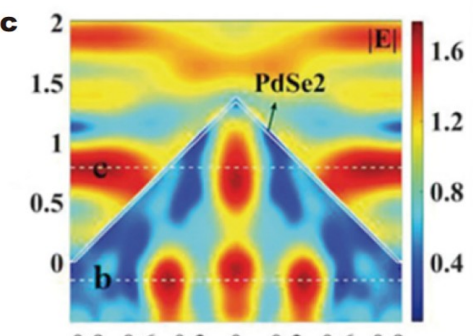

$\begin{array}{ccccccc}-0.9 & -0.6 & -0.3 & 0 & 0.3 & 0.6 & 0.9\end{array}$ $\mathrm{X}(\mu \mathrm{m})$

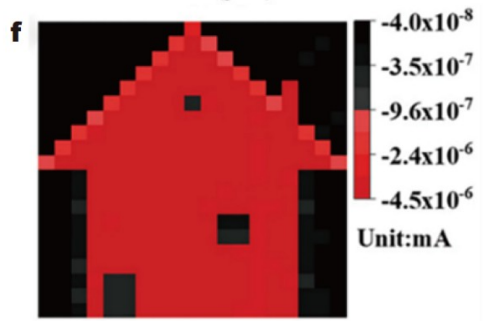

h

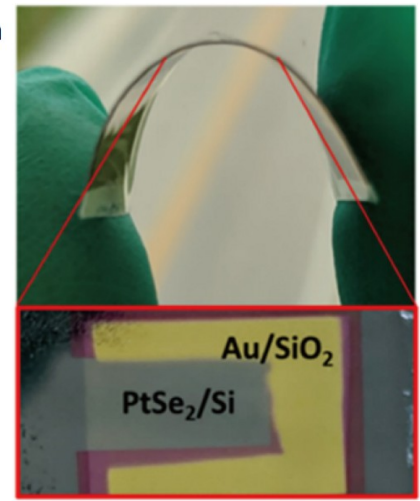

Figure 7 Group-ten TMDCs-based devices. (a) Schematic illustration of $\mathrm{PdSe}_{2} / \mathrm{Si}$ detector. Reprinted with permission from Ref. [95]. Copyright 2018, WILEY-VCH Verlag GmbH \& Co. KGaA, Weinheim. (b) Schematic diagram of the PdSe 2 /pyramid Si heterojunction photodetector. (c) Simulation of electric field energy distribution of the $\mathrm{PdSe}_{2} /$ pyramid Si with a wavelength of $980 \mathrm{~nm}$. (d) Digital photograph of the template "tree" and "house". The corresponding 2D current mapping of image: (e) tree under the illumination of $980 \mathrm{~nm}$ light, (f) house under the illumination of $1300 \mathrm{~nm}$ light. (b-f) are reprinted with permission from Ref. [96]. Copyright 2019, WILEY-VCH Verlag GmbH \& Co. KGaA, Weinheim. (g) The energy band diagram of p-Si, PtSe $e_{2}$ thin films with a thickness of 0.8, 1.4, 2.2, and $3.0 \mathrm{~nm}$. The $4 \mathrm{~L}(3.0 \mathrm{~nm}) \mathrm{PtSe}_{2}$ is semimetallic. Reprinted with permission from Ref. [98]. Copyright 2021, American Chemical Society. (h) The digital photograph of the $\mathrm{PtS}_{2} / \mathrm{Si}$ flexible device. Reprinted with permission from Ref. [99]. Copyright 2019, American Chemical Society.

CVD method. This as-made photocathode exhibited an outstanding conversion ability for direct solar-to-hydrogen $\left(\mathrm{H}_{2}\right)$ production due to the photoelectrochemical conversion. And the produced $\mathrm{H}_{2}$ would protect the heterostructure substrate from oxidation. What's more, they also demonstrated the atomiclayer-dependent band alignments at the $\mathrm{PtSe}_{2} / \mathrm{Si}$ interface. They found and figured out the work function of $\mathrm{PtSe}_{2}$ with different thicknesses and then constructed corresponding band diagrams for the $\mathrm{PtSe}_{2} / \mathrm{Si}$ heterostructure (as shown in Fig. 7g). In addition, the $\mathrm{PtSe}_{2} / \mathrm{Si}$ heterostructure is also reported to be used for flexible devices in another study [99]. The as-mentioned flexible device has not only excellent Schottky junction properties, such as high-current rectification ratio $\left(>10^{3}\right)$, small ideality factor $(n$ $\sim 1.9$ ), and temperature-dependent variation of Schottky barrier heights, but also good anti-deformation characteristics-after hundreds of bends, the photovoltaic characteristics of the device are still the same as before. Fig. 7h shows the flexible photovoltaic $2 \mathrm{D} \mathrm{PtSe}_{2} / \mathrm{Si}$ devices.

\section{Re-dichalcogenide-based devices}

$\mathrm{ReX}_{2}$ also have good performances especially in polarization detection. Liu et al. [100] demonstrated a $\mathrm{ReS}_{2}$ polarization sensitive photodetector on a $\mathrm{Si} / \mathrm{SiO}_{2}$ substrate. The detector showed an excellent polarization property with high responsivity of $\sim 10^{3} \mathrm{~A} \mathrm{~W}^{-1}$ and on/off ratio of $10^{5}$. Different from previous photodetectors, this $\mathrm{ReS}_{2} / \mathrm{SiO}_{2} / \mathrm{Si}$ photodetector did not require the use of extra components (gratings etc.) due to the anisotropic nature of the $\mathrm{ReS}_{2}$. In fact, the performances of $\mathrm{p}-\mathrm{n}$ junction made by $n-\mathrm{ReS}_{2} / \mathrm{p}-\mathrm{Si}$ are related to the thickness of $\mathrm{ReS}_{2}$; the p-n heterojunction with thin $\mathrm{ReS}_{2}$ layer showed weak photosensing performance but fast response, while the $p-n$ junction with thick multilayer $\mathrm{ReS}_{2}$ showed a larger photocurrent with an overall degradation of the response time. A mixed-thickness $\mathrm{ReS}_{2} / \mathrm{Si}$ showed a balance between responsivity and speed, with a high photoresponsivity of $33.47 \mathrm{~A} \mathrm{~W}^{-1}$ and a fast response time of $80 \mu \mathrm{s}$ [101]. In addition, the TMDC/TMDC heterostructures were also realized for $\mathrm{ReS}_{2}$. Ahn et al. [102] 
a
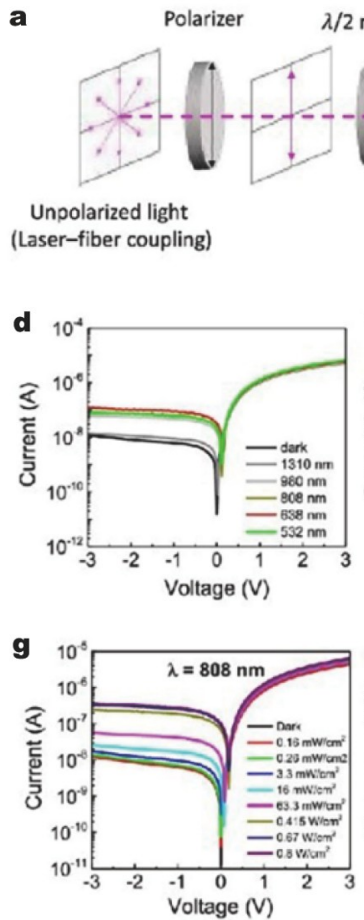
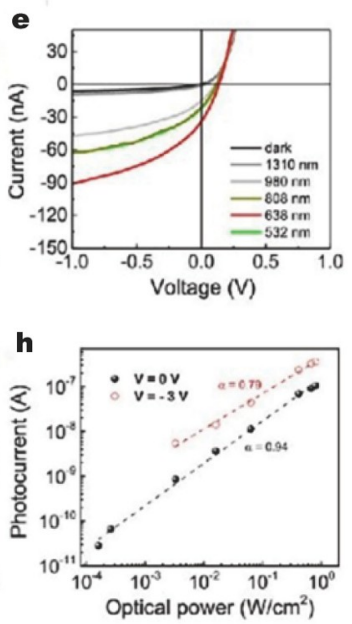
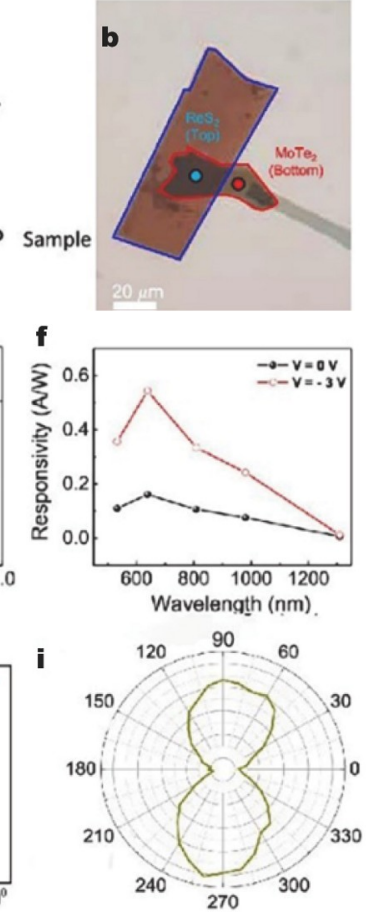
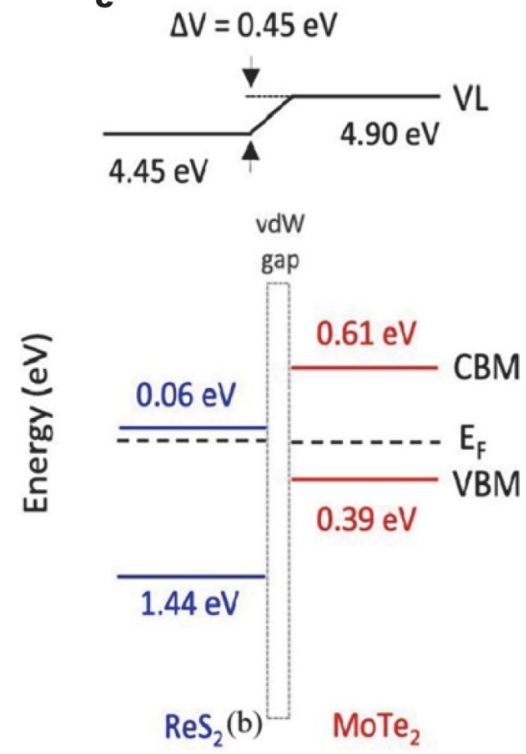

$\mathrm{ReS}_{2} / \mathrm{MoTe}_{2}$ Type-II

Figure 8 (a) Schematic illustration of the linear polarization-sensitive photodetection; (b) optical microscopy of $\mathrm{MoTe}_{2} / \mathrm{ReS}_{2}$ heterojunction; (c) energy level diagram of $\mathrm{ReS}_{2} / \mathrm{MoTe}_{2}$ van der Waal heterojunction, indicating the clear type-II band alignment. Dark and photoinduced current-voltage characteristics of a photodiode in (d) logarithmic scale and (e) linear scale under NIR (1310, 980, and $808 \mathrm{~nm})$, red (638 nm), and green (532 nm) light illuminations. (f) Two different mode responsivities as a function of illumination wavelength. (g) Dark and photoinduced current-voltage characteristics under different laser diode $(808 \mathrm{~nm})$ illumination powers. (h) Photocurrent versus optical power density. (i) Plot of linear polarization-sensitive normalized photocurrent changes in polar coordinates. Reprinted with permission from Ref. [102]. Copyright 2021, American Chemical Society.

fabricated a $\mathrm{MoTe}_{2} / \mathrm{ReS}_{2}$ heterostructure for a high performance photodiode with an additional function of linearly polarized photodetection (as shown in Fig. 8). The heterostructure photodiode shows an ideality factor of 1.3 , a rectification ratio of $10^{4}$, broad spectral response up to NIR light of $1310 \mathrm{~nm}$, and relatively high responsivity of $0.54 \mathrm{~A} \mathrm{~W}^{-1}$. There is no existing report about the $\mathrm{ReSe}_{2} / \mathrm{Si}$ and $\mathrm{ReTe}_{2} / \mathrm{Si}$ devices, though these two kinds of Re-dichalcogenide-based devices may possess more interesting performances.

\section{Brief summary}

Research activities of typical TMDC material-based photodetectors are emerging on a fast pace. This review only discussed some typical TMDC materials. Table 2 summarizes the performances of some representative TMDC-based devices mentioned in this paper. However, the large family of low-dimensional materials can be explored for developing more heterostructure photodetection devices. For example, other low-dimensional TMDCs such as $\mathrm{TiX}_{2}, \mathrm{CoX}_{2}, \mathrm{NiX}_{2}, \mathrm{SnX}_{2}, \mathrm{NbX}_{2}, \mathrm{TaX}_{2}(\mathrm{X}=\mathrm{S}$, Se, Te) [103-108], can be explored for developing new high-performance photodetectors. TMDCs are also advancing as multifunctional materials for future wearable electronic and optoelectronic devices as an inexpensive alternative to the costly manufactured semiconducting materials currently employed in the electronics industry. Low-dimensional atomic layered material-based hybrid van de Waals heterostructures hold great potential for self-powered flexible photodetectors in the healthcare industry especially for biomedical imaging, point-ofcare wearable biosensors for monitoring vital signs and meta- bolic parameters, and for patients. In addition, TMDCs are very promising nanomaterials for developing high-performance flexible photodetectors and for integration of such devices into the next generation of wearable technologies. The integration of cross-dimensional heterostructure TMDC photodetectors with other electronic, photonic and optoelectronic functional devices remains challenging.

\section{Black phosphorus devices}

Besides graphene and TMDCs, BP is another kind of lowdimensional material that attracts much attention. BP is a rare allotrope of phosphorus with direct bandgap and its layers stack together via van der Waals interaction. The bandgap of BP is thickness-dependent, which decreases from $1.7 \mathrm{eV}$ (for monolayer $\mathrm{BP}$ ) to $0.3 \mathrm{eV}$ (for bulk $\mathrm{BP}$ ) [109], covering the band from visible to MIR, just filling the gap of graphene and TMDCs. BP also has tunable bandgap and moderate switching ratio, good regulation and other excellent characteristics. For the perspective at a atomic level, each phosphorus atom is connected with three phosphorus atoms, causing BP crystal to form two special directions: the armchair and the zigzag directions along the $x$ and $y$ axes, respectively. So BP has prominent in-plane anisotropy. This special structure brings anisotropic electronic band dispersion, hence bringing anisotropic electronic and optoelectronic properties. In addition, for single/few-layered BP, the hole mobility can reach as high as $1000 \mathrm{~cm}^{2} \mathrm{~V}^{-1} \mathrm{~s}^{-1}$. The most unique property of BP is the strong in-plane anisotropy. In its $D_{2 h}$ point group orthorhombic crystal system, the carriers along the longitudinal sawtooth pattern is ten times more effective than 
Table 2 Comparison of the critical parameters for some TMDC-based photodetectors

\begin{tabular}{|c|c|c|c|c|c|}
\hline Device structure & Wavelength $(\mathrm{nm})$ & Responsivity $\left(\mathrm{A} \mathrm{W}^{-1}\right)$ & Detectivity (Jones) & Response time $(\mu s)$ & Ref. \\
\hline Few-layer $\mathrm{MoS}_{2} / \mathrm{Si}$ heterojunction & $450-1050$ & 0.3 & $10^{13}$ & 3 & {$[24]$} \\
\hline $\mathrm{MoS}_{2}$ (S defects) & $445-4700$ & $5.07 \times 10^{-3}$ & $1.55 \times 10^{9}$ & - & {$[66]$} \\
\hline $\mathrm{p}-\mathrm{Si} / \mathrm{n}-\mathrm{MoS}_{2}$ heterojunction & $350-950$ & 8.75 & $1.4 \times 10^{12}$ & $10 / 19$ & {$[20]$} \\
\hline $\mathrm{MoS}_{2} / \mathrm{P}(\mathrm{VDF}-\mathrm{TrFe}) / \mathrm{SiO}_{2} / \mathrm{Si}$ & $450-1550$ & 2570 & $2.2 \times 10^{12}$ & $1.8 \times 10^{3}$ & {$[67]$} \\
\hline 3D RGO-MoS 2 /pyramid-Si & $350-4500$ & 21.8 & $3.8 \times 10^{15}$ & - & {$[68]$} \\
\hline $2 \mathrm{H}-\mathrm{MoS}_{2} / 1 \mathrm{~T} @ 2 \mathrm{H}-\mathrm{MoS}_{2} / \mathrm{SiO}_{2} / \mathrm{Si}$ & Green light & 1917 & $\sim 10^{11}$ & - & {$[73]$} \\
\hline Graphene/MoSe $2 / \mathrm{Si}$ & $365-1310$ & 0.27 & $7.13 \times 10^{10}$ & 0.3 & {$[81]$} \\
\hline $\mathrm{MoSe}_{2} / \mathrm{SiO}_{2} / \mathrm{Si}$ & 785 & 238 & $7.6 \times 10^{11}$ & - & {$[82]$} \\
\hline $\mathrm{p}-\mathrm{MoSe}_{2} / \mathrm{n}-\mathrm{Si}$ junction & $405-2500$ & 0.52 & $6.7 \times 10^{9}$ & $18 / 10$ & {$[83]$} \\
\hline $\mathrm{MoTe}_{2}$ & 685 & 6 & - & 160 & {$[87]$} \\
\hline $1 \mathrm{~T}-\mathrm{MoTe}_{2} / 2 \mathrm{H} \mathrm{MoTe} e_{2}$ & 980 & - & - & 99 & [89] \\
\hline n-MoTe $2 / n-S i$ junction & $300-1800$ & 0.19 & $6.8 \times 10^{13}$ & 0.15 & {$[88]$} \\
\hline $\mathrm{WSe}_{2} / \mathrm{ITO}$ & $370-1064$ & 0.92 & - & $9 \times 10^{5}$ & {$[90]$} \\
\hline $\mathrm{WSe}_{2} / \mathrm{Si}$ junction & $390-1088$ & 0.12 & - & - & {$[91]$} \\
\hline Wafer-scale-WS $\mathrm{W}_{2} / \mathrm{Si}$ & $350-850$ & $4 \times 10^{-3}$ & $1.5 \times 10^{10}$ & 1.2 & {$[84]$} \\
\hline $\mathrm{Ag}-\mathrm{WS}_{2} / \mathrm{Si}$ & $390-670$ & 2.09 & $6.6 \times 10^{11}$ & $2 \times 10^{5}$ & {$[86]$} \\
\hline $\mathrm{WS}_{2} / \mathrm{Si}$ array & $200-3043$ & 8.3 & $4.6 \times 10^{14}$ & $16 / 29$ & {$[85]$} \\
\hline $\mathrm{PdSe}_{2} / \mathrm{Si}$ & $200-3044$ & 0.3 & $\sim 10^{13}$ & $38 / 44$ & [95] \\
\hline $\mathrm{PdSe}_{2} /$ pyramid Si & $980-1650$ & 0.45 & $9.97 \times 10^{13}$ & - & [96] \\
\hline $\mathrm{ME}-\mathrm{PdSe} \mathrm{e}_{2} / \mathrm{Si}$ & $400-1200$ & 47.2 & $8.13 \times 10^{9}$ & $9.2 / 17.3$ & [97] \\
\hline $\mathrm{ReS}_{2} / \mathrm{SiO}_{2} / \mathrm{Si}$ & $450-800$ & - & - & 160 & {$[100]$} \\
\hline $\mathrm{p}-\mathrm{ReS}_{2} / \mathrm{Si}$ & - & 33.47 & - & 90 & {$[101]$} \\
\hline $\mathrm{MoTe}_{2} / \mathrm{ReS}_{2}$ & $532-1310$ & 0.54 & $10^{10}$ & 2.4 & {$[102]$} \\
\hline
\end{tabular}

that along the transverse structure. This property makes it possible to use BP for the design of new types of electronic and photonic devices.

\section{$B P / S i$ waveguide photodetectors}

However, the studies of $\mathrm{BP} / \mathrm{Si}$ heterojunctions are scarcely reported. A few related reports are the studies of BP and silicon waveguides. These hybrid Si/BP waveguide photodetectors have similar constructions that are mostly focused on the NIR-MIR region (see Fig. 9a, b). For the hybrid BP/Si waveguide photodetector, the anisotropy of BP thin film also plays an important role in the electronic and optical properties of the photodetectors. Different thicknesses and different orientations will influence the photodetectors' performances. For example, Yin et al. [110] demonstrated a silicon photonic integrated BP detector operating at $2 \mu \mathrm{m}$ wavelength. Then, they calculated the light absorption coefficient and electric-field profiles of BP with different thicknesses at different orientation angles (the $\theta$ is 0 when the orientation is along with the armchair direction) (see Fig. 9c, d). In this BP/Si waveguide heterostructure, BP film can cover the top and side walls of SOI ridge waveguide well, thus enhancing the light absorption of the transverse electro fundamental mode $\left(\mathrm{TE}_{0}\right)$ in the ridge waveguide. The device exhibited a responsivity of $306.7 \mathrm{~mA} \mathrm{~W}^{-1}$ and a $3 \mathrm{~dB}$ bandwidth of $1.33 \mathrm{GHz}$ at a low bias voltage of $0.4 \mathrm{~V}$. In another $\mathrm{BP} / \mathrm{Si}$ waveguide heterostructure [111], the BP film was integrated on the grating coupler, and the silicon waveguide and grating coupler ensured the longitudinal light propagation and out-ofplane coupling, thus enhancing the interaction between light and BP. In this report, they also found the responsivity would be influenced by the thickness and orientation (as shown in Fig. 9f, g). In addition, the photoresponse would be tuned by gate and drain bias (as shown in Fig. 9e), indicating the changes in band structures.

\section{Protection to $B P$}

Although BP has shown excellent optoelectronic properties, its instability has become one of the greatest concerns and hinders its further development. Some studies have been shown that BP flakes will quickly degrade and lose their electrical properties after a few hours/days at atmosphere conditions. Researchers have tried some strategies to enhance the stability of BP, including covering a passive layer ( $\mathrm{h}-\mathrm{BN}, \mathrm{Al}_{2} \mathrm{O}_{3}$, etc.) to protect the BP layer [112]. Recently, the group of Zhang [113] tried a new material, hydrophobic polyionic liquid poly(1-hexyl-3vinylimidazolium) hexafluorophosphate salt (PIL-TFSI), as the protecting layer to form a self-healable photodetector. In this PIL/BP photodetector, the PIL-TFSI layer provided a significantly enhanced stability as well as a fluorination to BP. In addition, this detector showed a self-healing capability due to the self-healing nature of PIL-TFSI. The typical on/off signals can still be detected in this photodetector even after 50 cycles. Herein, the organic reagent PIL-TFSI may be a promising material to enhance the stability of BP-based devices in future applications.

\section{Brief summary}

BP-based devices have shown excellent optoelectronic properties; taking advantage of BP's adjustable bandgap property, they exhibit excellent performances in the short-to-mid IR range. Especially when combined with $\mathrm{Si}$, the devices not only have the high-speed characteristic of Si-based detector, but also exhibit 


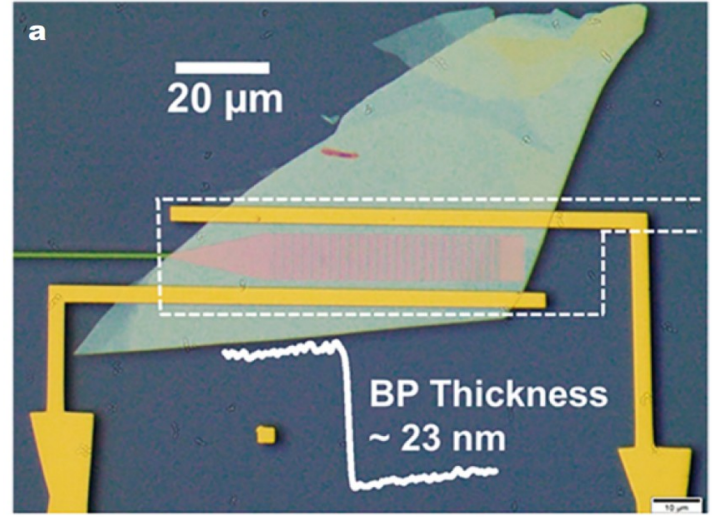

C

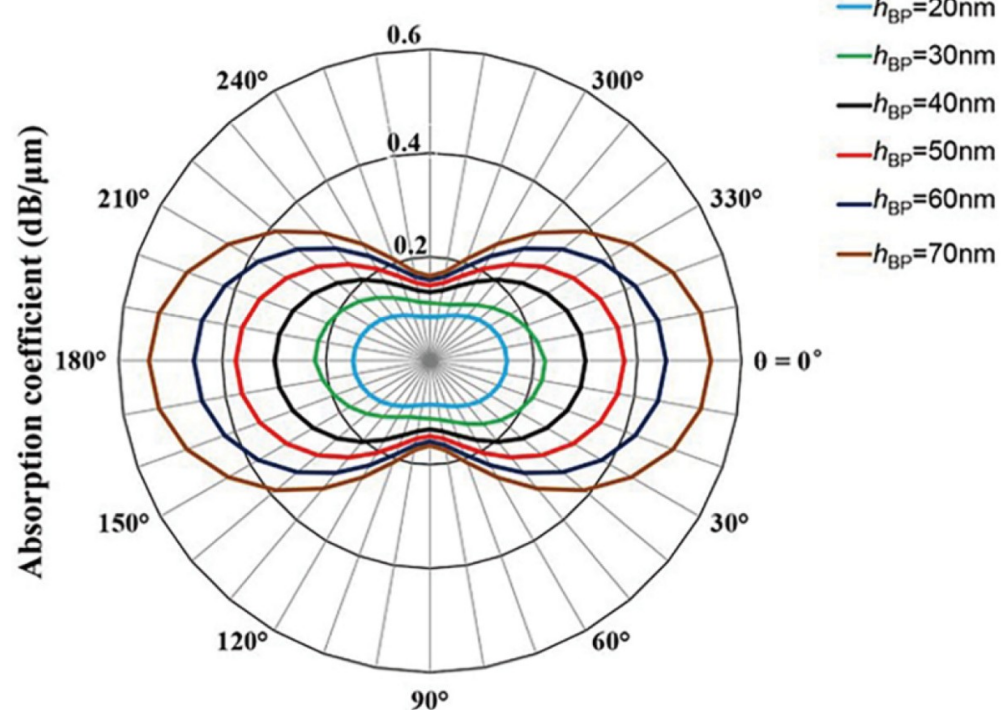
$I_{p h}(\mu \mathrm{A})$

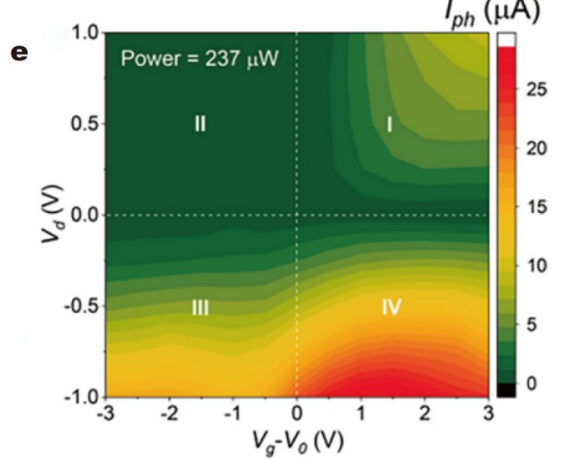

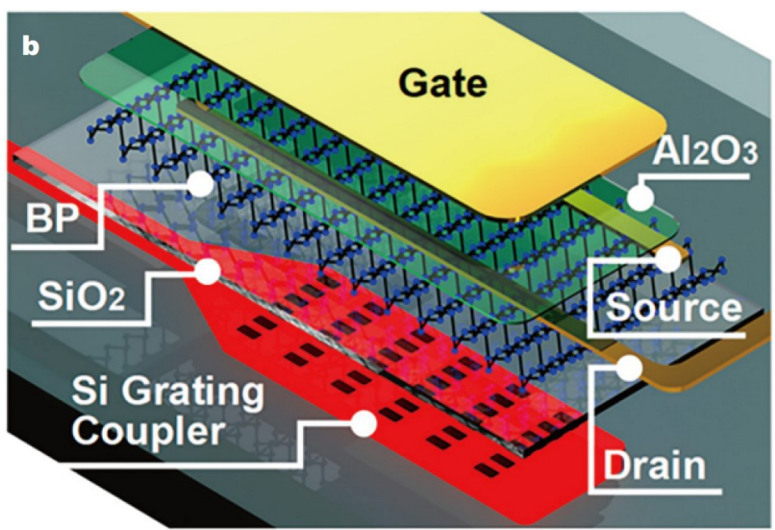

d
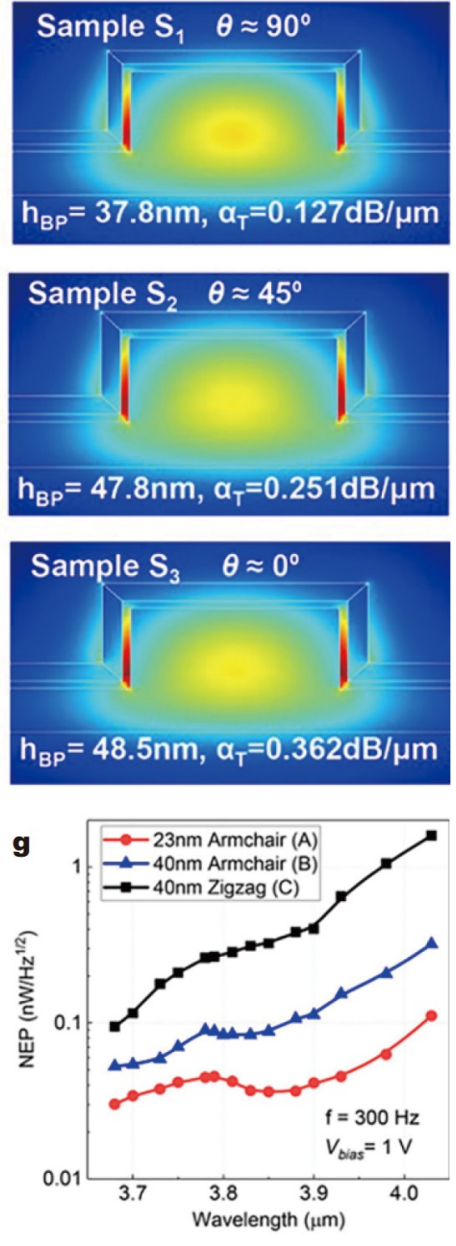

Figure 9 (a) Microscopy image of a BP photodetector on Si waveguide. (b) Schematics of the waveguide-integrated on-chip system with BP photodetector. (c) Light absorption coefficient $\alpha$ as the BP orientation angle $\theta$ varies from $0^{\circ}$ to $360^{\circ}$. The thickness of BP is $20,30,40,50,60,70 \mathrm{~nm}$. (d) Calculated electric field profiles of the $\mathrm{TE}_{0}$ mode supported in the hybrid silicon/BP waveguides at $2 \mu \mathrm{m}$. (e) Contour plot of gate and drain-dependent photocurrent under $237 \mu \mathrm{W}$ illumination at $3.78 \mu \mathrm{m}$. The optimized photocurrent is obtained with negative drain bias. (f) Spectral responsivities and (g) NEPs of the BP/Si waveguides with different thicknesses and orientations. (a, b, e-g) are reprinted with permission from Ref. [111]. Copyright 2018, American Chemical Society. (c, d) are reprinted with permission from Ref. [110]. Copyright 2019, WILEY-VCH Verlag GmbH \& Co. KGaA, Weinheim.

the property of anisotropy which Si-based detectors donot possess. However, for better performances of the BP/Si detector, the stability will be the focus to be studied.

\section{OTHER LOW-DIMENSIONAL}

\section{HETEROSTRUCTURE-BASED DEVICES}

Apart from Si-based heterostructure-based photodetectors, ger- manium (Ge) heterostructure is also employed to fabricate IR photodetectors. Ge has distinct properties, such as high light absorption, low cost and easy fabrication process, and narrower bandgap $(0.66 \mathrm{eV})$ [114]. Graphene/Ge heterostructure detectors have unique characteristics. Zeng et al. [62] fabricated an SLG/ Ge Schottky heterojunction for the IR light sensing. The graphene/Ge photodetectors were sensitive to IR light with the 
responsivity of $51.8 \mathrm{~mA} \mathrm{~W}^{-1}$, detectivity of $1.38 \times 10^{10}$ Jones, and on/off ratio of $2 \times 10^{4}$. It is worth noting that the photovoltaic IR detector exhibited a peak sensitivity at $1400 \mathrm{~nm}$, corresponding to the intrinsic absorption of $\mathrm{Ge}$, which means a good spectral selectivity. In addition, the SLG/Ge detectors exhibited good reproducibility and long-term stability. In fact, in Huang's report [11], the graphene $/ \mathrm{HfO}_{2} / \mathrm{Ge}$ and graphene $/ \mathrm{HfO}_{2} /$ a- $\mathrm{MoS}_{2}$ photodetectors were also studied, and the graphene/ $\mathrm{HfO}_{2} / \mathrm{Ge}$ photodetectors exhibited high responsivity of $9.03 \mathrm{~A} \mathrm{~W}^{-1}$ (at $0.1 \mathrm{~V}$ bias), fast response speed of $\tau_{\text {rise }}=11 \mu \mathrm{s}$, $\tau_{\text {decay }}=38 \mu \mathrm{s}$, which are superior to previous graphene $/ \mathrm{Ge}$ devices. And the graphene/ $\mathrm{HfO}_{2} / \mathrm{a}-\mathrm{MoS}_{2}$ photodetectors showed sensitivities to $473-2712 \mathrm{~nm}$ light with responsivity of $5.36 \mathrm{~A} \mathrm{~W}^{-1}$ and detectivity of $1.57 \times 10^{9}$ Jones. These excellent performances suggested the graphene-based devices may have great potential for future IR detection application. Mahyavanshi et al. [115] demonstrated the $\mathrm{MoS}_{2} / \mathrm{Ge}$ heterostructure photodetectors. At different bias voltage, the detector showed distinct photoresponsivity for UV, visible, and NIR light. And the response to UV light is a significant breakthrough for $\mathrm{MoS}_{2}$ based photodetectors. On the other hand, some scientists have tried to directly fabricate the heterostructure of $\mathrm{MoS}_{2}$ thin film and substrate. $\mathrm{MoTe}_{2} / \mathrm{Ge}$ heterostructure-based devices are also exploited for NIR detection. Chen et al. [116] transferred $\mathrm{MoTe}_{2}$ nanosheet on an undoped $\mathrm{Ge}$ substrate with 30 -nm-thick $\mathrm{Al}_{2} \mathrm{O}_{3}$ as an isolating layer. The as-made photodetector exhibited excellent performances with very high responsivity of $12,460 \mathrm{~A} \mathrm{~W}^{-1}$ and specific detectivity of $3.3 \times 10^{12}$ Jones. However, the response speed is relatively low with a specific time of $5 \mathrm{~ms}$, and the reverse current at dark is large due to the low barrier between $\mathrm{MoTe}_{2}$ and $\mathrm{Ge}$. So a $\mathrm{GeO}_{2}$ was introduced between the $\mathrm{MoTe}_{2}$ and $\mathrm{Ge}$ [117], and the reverse current was reduced by more than four orders of magnitude at a cost of reduced responsivity and detectivity (specific value of $15.6 \mathrm{~A} \mathrm{~W}^{-1}$ and $4.86 \times 10^{11}$ Jones, respectively).

Gallium nitride ( $\mathrm{GaN}$ ) has also become a hot material to be used with $\mathrm{MoS}_{2}$ to develop hybrid photodetectors due to their low lattice mismatch. Zhuo et al. [118] made a $\mathrm{PtSe}_{2} / \mathrm{GaN}$ photodetector which was in-situ fabricated by synthesis of vertically standing low-dimensional $\mathrm{PtSe}_{2}$ film on $\mathrm{n}-\mathrm{GaN}$ substrate. The $\mathrm{PtSe}_{2} / \mathrm{GaN}$ heterojunction device has excellent photoresponse properties under illumination by DUV light. This photodetector had a high responsivity of $193 \mathrm{~mA} \mathrm{~W}^{-1}$, an ultrahigh specific detectivity of $3.8 \times 10^{14}$ Jones, $I_{\text {on }} / I_{\text {off }}$ ratio of $\sim 10^{8}$, as well as short rise and decay times of $45 / 102 \mu$ s. They also fabricated the self-powered few-layered $\mathrm{MoS}_{2} / \mathrm{GaN}$ p-n heterojunction photodetector which could work in DUV [119]. In order to develop the $\mathrm{MoS}_{2} / \mathrm{GaN}$ p-n heterojunction photodetector, the $\mathrm{MoS}_{2}$ thin films were transferred onto a $\mathrm{GaN}$ substrate where the Au electrode was deposited on the $\mathrm{MoS}_{2}$ film and the $\mathrm{Ni} / \mathrm{Au}$ electrode was deposited on the $\mathrm{GaN}$ via the EBE method. The $\mathrm{MoS}_{2} / \mathrm{GaN}$ photodetector exhibited a current $I_{\mathrm{on}} /$ $I_{\text {off }}$ ratio of $10^{5}$ at an illumination intensity of $2.4 \mu \mathrm{W} \mathrm{cm}{ }^{-2}$ at zero bias voltage, indicating the photodetectors could work in the UV region without ultra power. The $\mathrm{MoS}_{2} / \mathrm{GaN}$ photodetectors exhibited photoresponsivity of $187 \mathrm{~mA} \mathrm{~W}^{-1}$, detectivity of $2.34 \times 10^{13}$ Jones, fast response speeds of $46.4 \mu$ s at $0 \mathrm{~V}$ bias. Recently, Li et al. [13] tried to fabricate monolayer $\mathrm{MoS}_{2} /$ GaN-based devices and introduced tensile strain by depositing ultrathin $\mathrm{Al}_{2} \mathrm{O}_{3}$ layer $(3 \mathrm{~nm})$ to improve photovoltaic performances. As a result, the monolayer $\mathrm{MoS}_{2} / \mathrm{GaN} / \mathrm{Al}_{2} \mathrm{O}_{3}$ heterostructure-based photodetector achieved higher responsivity of $24.6 \mathrm{~A} \mathrm{~W}^{-1}$, and their $G$ and EQE are as high as $520 \%$ and $8381 \%$, respectively. On the other hand, strain-adjusting of the bandgap of $\mathrm{MoS}_{2}$ using designed pattern substrate is also an effective way to improve the performances of $\mathrm{MoS}_{2} / \mathrm{GaN}$-based photodetector. Liu et al. [120] designed orthohexagonal GaN arrays on a sapphire substrate, and then transferred $\mathrm{MoS}_{2}$ on the pattern GaN substrate (PGS) with the help of polymethyl methacrylate; at the same time, they transferred $\mathrm{MoS}_{2}$ on the flat GaN substrate (FGS) for comparison (Fig. 10). Under the same conditions, the $\mathrm{MoS}_{2} / \mathrm{PGS}$-based photodetector showed higher photocurrent and higher responsivity; the NEP decreased to 3.88 $\times 10^{-13} \mathrm{~W} \mathrm{~Hz}^{-1 / 2}$, and $D^{*}$ increased to $5.6 \times 10^{8}$ Jones when
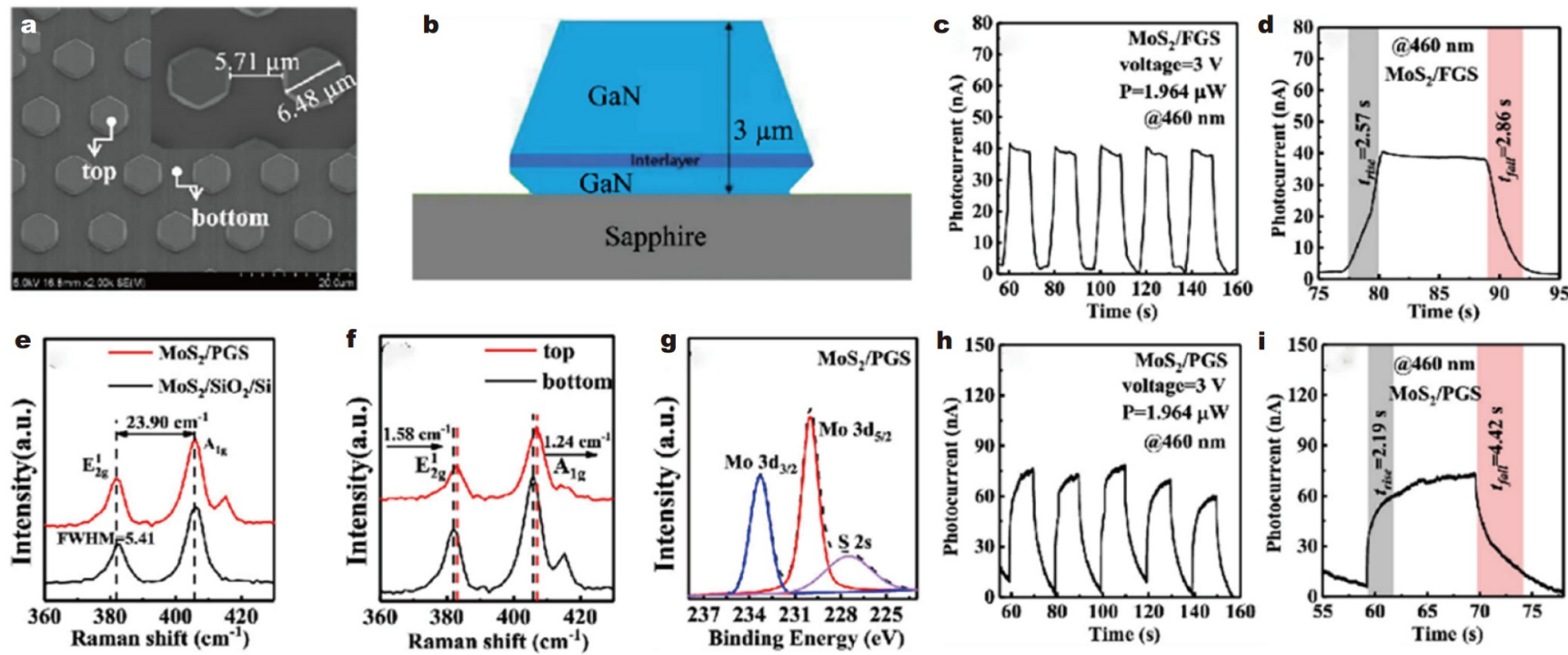

Figure 10 (a) Scanning electron microscopy (SEM) image of the PGS, where the inset shows the size of the patterned array; (b) schematic cross-sectional view of the PGS; switching characteristics and rise/fall times of (c, d) $\mathrm{MoS}_{2} / \mathrm{FGS}$ and (h, i) MoS 2 /PGS photodetectors under 460 nm laser illumination at 3 V; (e, f) Raman spectra of the $\mathrm{MoS}_{2}$ film (e) before and after transfer and (f) at the top and bottom of the patterned array; (g) core-level X-ray photoelectron spectroscopy (XPS) spectrum of $\mathrm{MoS}_{2}$ /PGS. Reprinted with permission from Ref. [120]. Copyright2021, American Chemical Society. 
compared with the $\mathrm{MoS}_{2} / \mathrm{FGS}$-based devices.

Moreover, the heterostructures of low-dimensional materials and other conventional substrates also have different performances. Nasr et al. [121] successfully combined $\mathrm{MoS}_{2}$ and glass to fabricate a low-power photodetector. They first synthesized atomically thin $\mathrm{MoS}_{2}$ on transparent glass substrate at a relatively low temperature via metal organic CVD (MOCVD), then grew high- $k$ (dielectric constant) and ultra-thin dielectric $\mathrm{Al}_{2} \mathrm{O}_{3}$ layer as the top-gate dielectric to accomplish the low-power phototransistors. The as-made $\mathrm{MoS}_{2}$-based photodetectors can be excited by low-intensity light source instead of usual laser excitation. It is worth noting that the energy consumption of the $\mathrm{MoS}_{2}$-based photodetector is as low as about 100 pico Joules, which shows promise to realize the energy-efficient lowdimensional glasstronic technology. Xu et al. [122] successfully fabricated a $\mathrm{MoS}_{2} / \mathrm{GaAs}$ heterostructure as a self-driven photodetector. And the $\mathrm{MoS}_{2} / \mathrm{GaAs}$ heterostructure-based photodetector showed excellent optoelectronic performances with responsivity of $321 \mathrm{~mA} \mathrm{~W}^{-1}$ and detectivity of $3.5 \times 10^{13}$ Jones, respectively. To further improve the performances of the detector, they introduced an h-BN thin film to constitute a $\mathrm{MoS}_{2} / \mathrm{h}-\mathrm{BN} / \mathrm{GaAs}$ sandwich structure which could effectively suppress the dark current. And the responsivity was increased to $419 \mathrm{~mA} \mathrm{~W}^{-1}$ as well as the detectivity was increased to $1.9 \times$ $10^{14}$ Jones.

\section{SUMMARY AND OUTLOOK}

Optoelectronic devices based on heterostructures of 2D materials have exhibited novel phenomena and achieved many high performances. It can be said that we have achieved great success in the research of $2 \mathrm{D}$ material/conventional substrate heterostructures. We herein reviewed the recent progress in the performances of cross-dimensional material-based photonic and optoelectronic devices, mainly focused on several typical 2D materials, including graphene, TMDCs, and BP. In recent years, tremendous advances have been made in the design of crossdimensional heterostructures, such as larger spectrum range, faster speed, and better optoelectronic performances. For example, the spectral range of $\mathrm{MoS}_{2} / \mathrm{Ge}$ is broadened to IR region. The patterned $\mathrm{GaN}$ helps a lot to improve the performances of $\mathrm{MoS}_{2}$-based photodetectors. However, the promising and the one which has attracted the most attention, is the combination of 2D material and silicon. Some kinds of Si-based cross-dimensional heterojunction devices can break the limitations of the original low-dimensional materials and obtain higher responsivities and detectivities. In addition, the Si-based low-dimensional heterostructure devices are more likely to show high speed characteristics, providing a certain possibility for future communication circuits. Most important of all, silicon is the conventional material widely used in integrated circuits for that the cost of silicon is relatively cheap and the related technology is the most mature. Therefore, the Si substrate-based cross-dimensional heterostructure devices have the most potential to be embedded in modern large-scale integrated circuits as well as with a commercial potential. On the other hand, the low-dimensional materials can make up for some shortcomings of silicon materials, enhancing the light absorption for silicon even providing an opportunity for the smaller and smaller channel length in future. Herein, in our opinion, the future focus of cross-dimensional heterostructure is Si-based heterostructure. And there are some possible directions just for reference: (1) find more kinds of low-dimensional materials to probe different possibilities of the Si-based heterostructure. Out of a sea of low-dimensional materials, there are always better low-dimensional materials that are more suitable for siliconbased heterostructure systems to be found and synthesized. (2) Dig deep in the common materials. The band structures of these heterostructures can be adjusted by the methods of introducing defects of low-dimensional materials, such as adjusting doping concentrations of $\mathrm{Si}$ substrate, and changing the interface states between the heterostructure. (3) Utilize the modern technologies, especially for these large-area $2 \mathrm{D}$ materials. The mature technologies would help the 2D/3D heterostructures form photodetection arrays, and even provide a potential compatible to large-scale integrated circuits.

Despite the novel phenomena shown and many high performances gained in the cross-dimensional heterostructures, there are still some problems and challenges. There are some fields requiring additional improvement: (1) processability and practical application. Most of reported studies are manipulated by manual system, which causes a hindrance for the industrialization and mass production. (2) Key parameters. Most of present devices can only enhance one or two aspects of the key parameters. Some cross-dimensional structure-based devices even have exhibited worse performances than pristine independent low-dimensional material-based devices. (3) Small synthesis area, except for several kinds of low-dimensional materials. Most low-dimensional materials are obtained with small scale, so it is difficult to obtain their arrays, hindering fast imaging that can be used in practical applications. Herein, more efforts need to be cast to improve full aspects of the devices. It is a long-term process for all stuff involved, not only for the researchers of lowdimensional materials to get high-quality crystalline and probe the possibilities of new cross-dimensional heterostructures, but also for the practitioners of conventional technologies to perfect various processes of the optoelectronic devices, as well as for the theoretical physicists to provide a broader platform to design more band structures with demand, even for the governor of related department, the ordinary people to pay more attention to low-dimensional materials to support the development of related industries. There are many significant challenges in producing low-cost, large-scale, high-performance photodetector devices for commercial applications, and therefore factors such as optimization of the photoresponse, reproducibility, and longterm environmental stability of photodetector devices used for photodetectors need much more attention.

Received 21 October 2021; accepted 17 December 2021; published online 30 December 2021

1 Novoselov KS, Geim AK, Morozov SV, et al. Electric field effect in atomically thin carbon films. Science, 2004, 306: 666-669

2 Mueller NS, Okamura Y, Vieira BGM, et al. Deep strong light-matter coupling in plasmonic nanoparticle crystals. Nature, 2020, 583: 780784

3 Kim H, Uddin SZ, Lien DH, et al. Actively variable-spectrum optoelectronics with black phosphorus. Nature, 2021, 596: 232-237

4 He W, Hong T, Wang D, et al. Low carrier concentration leads to high in-plane thermoelectric performance in n-type SnS crystals. Sci China Mater, 2021, 64: 3051-3058

5 Zheng Z, Chen $\mathrm{P}$, Lu J, et al. Self-assembly $\mathrm{In}_{2} \mathrm{Se}_{3} / \mathrm{SnSe}_{2}$ heterostructure array with suppressed dark current and enhanced photosensitivity for weak signal. Sci China Mater, 2020, 63: 1560-1569

6 Zhao X, Lin Z, Wang Y, et al. Label-free self-referenced sensing of 
living cells by terahertz metamaterial-based reflection spectroscopy. Biomed Opt Express, 2019, 10: 1196-1206

7 Wang Y, Wang H, Gali SM, et al. Molecular doping of 2D indium selenide for ultrahigh performance and low-power consumption broadband photodetectors. Adv Funct Mater, 2021, 31: 2103353

8 AlAloul M, Rasras M. Plasmon-enhanced graphene photodetector with CMOS-compatible titanium nitride. J Opt Soc Am B, 2021, 38: 602

9 Wan $\mathrm{X}, \mathrm{Xu} \mathrm{Y}$, Guo $\mathrm{H}$, et al. A self-powered high-performance graphene/silicon ultraviolet photodetector with ultra-shallow junction: Breaking the limit of silicon? npj 2D Mater Appl, 2017, 1: 4

10 Duan B, Li M, Dong Z, et al. New super-junction LDMOS breaking silicon limit by multi-ring assisted depletion substrate. IEEE Trans Electron Devices, 2019, 66: 4836-4841

11 Huang Z, Liu J, Zhang T, et al. Interfacial gated graphene photodetector with broadband response. ACS Appl Mater Interfaces, 2021, 13: 22796-22805

12 Ran S, Glen TS, Li B, et al. The limits of electromechanical coupling in highly-tensile strained germanium. Nano Lett, 2020, 20: 3492-3498

13 Li Z, Luo J, Hu S, et al. Strain enhancement for a $\mathrm{MoS}_{2}$-on-GaN photodetector with an $\mathrm{Al}_{2} \mathrm{O}_{3}$ stress liner grown by atomic layer deposition. Photon Res, 2020, 8: 799

14 Shi K, Li J, Xiao Y, et al. High-response, ultrafast-speed, and selfpowered photodetection achieved in InP@ZnS- $\mathrm{MoS}_{2}$ phototransistors with interdigitated Pt electrodes. ACS Appl Mater Interfaces, 2020, 12: 31382-31391

15 Jia C, Zhao X, Lai YH, et al. Highly flexible, robust, stable and high efficiency perovskite solar cells enabled by van der Waals epitaxy on mica substrate. Nano Energy, 2019, 60: 476-484

16 Dang ZM, Zheng MS, Zha JW. 1D/2D carbon nanomaterial-polymer dielectric composites with high permittivity for power energy storage applications. Small, 2016, 12: 1688-1701

17 Wang H, Naghadeh SB, Li C, et al. Enhanced photoelectrochemical and photocatalytic activities of CdS nanowires by surface modification with $\mathrm{MoS}_{2}$ nanosheets. Sci China Mater, 2018, 61: 839-850

$18 \mathrm{Li} \mathrm{S}, \mathrm{He} \mathrm{Z}$, Ke Y, et al. Ultra-sensitive self-powered photodetector based on vertical $\mathrm{MoTe}_{2} / \mathrm{MoS}_{2}$ heterostructure. Appl Phys Express, 2020, 13: 015007

19 Hou H, Zeng X, Zhang X. 2D/2D heterostructured photocatalyst: Rational design for energy and environmental applications. Sci China Mater, 2020, 63: 2119-2152

20 Dhyani V, Das S. High-speed scalable silicon- $\mathrm{MoS}_{2} \mathrm{P}-\mathrm{N}$ heterojunction photodetectors. Sci Rep, 2017, 7: 44243

21 Xia Y, Cheng B, Fan J, et al. Near-infrared absorbing 2D/3D ZnIn $\mathrm{S}_{4} /$ $\mathrm{N}$-doped graphene photocatalyst for highly efficient $\mathrm{CO}_{2}$ capture and photocatalytic reduction. Sci China Mater, 2020, 63: 552-565

22 Riedl C, Coletti C, Iwasaki T, et al. Quasi-free-standing epitaxial graphene on $\mathrm{SiC}$ obtained by hydrogen intercalation. Phys Rev Lett, 2009, 103: 246804

23 An X, Liu F, Jung YJ, et al. Tunable graphene-silicon heterojunctions for ultrasensitive photodetection. Nano Lett, 2013, 13: 909-916

24 Wang L, Jie J, Shao Z, et al. $\mathrm{MoS}_{2} / \mathrm{Si}$ heterojunction with vertically standing layered structure for ultrafast, high-detectivity, self-driven visible-near infrared photodetectors. Adv Funct Mater, 2015, 25: 2910-2919

25 Mortazavi Zanjani SM, Holt M, Sadeghi MM, et al. 3D integrated monolayer graphene-Si CMOS RF gas sensor platform. npj 2D Mater Appl, 2017, 1: 36

26 Nicolosi V, Chhowalla M, Kanatzidis MG, et al. Liquid exfoliation of layered materials. Science, 2013, 340: 1226419

27 Buzaglo M, Bar IP, Varenik M, et al. Graphite-to-graphene: Total conversion. Adv Mater, 2017, 29: 1603528

28 Hernandez Y, Nicolosi V, Lotya M, et al. High-yield production of graphene by liquid-phase exfoliation of graphite. Nat Nanotech, 2008, 3: $563-568$

29 Wang S, Tao B, Yu S, et al. Insight into the liquid-phase exfoliation to prepare BN nanosheets. Mater Lett, 2020, 269: 127644

30 Han GQ, Liu YR, Hu WH, et al. WS 2 nanosheets based on liquid exfoliation as effective electrocatalysts for hydrogen evolution reac- tion. Mater Chem Phys, 2015, 167: 271-277

31 Yang G, Huang H, Xiao Z, et al. A novel strategy for liquid exfoliation of ultrathin black phosphorus nanosheets. j Biomed nanotechnol, 2020, 16: 548-552

32 Teng C, Xie D, Wang J, et al. Ultrahigh conductive graphene paper based on ball-milling exfoliated graphene. Adv Funct Mater, 2017, 27: 1700240

33 Han G, Zhao X, Feng Y, et al. Highly flame-retardant epoxy-based thermal conductive composites with functionalized boron nitride nanosheets exfoliated by one-step ball milling. Chem Eng J, 2021, 407 127099

34 Ji H, Hu S, Jiang Z, et al. Directly scalable preparation of sandwiched $\mathrm{MoS}_{2}$ /graphene nanocomposites via ball-milling with excellent electrochemical energy storage performance. Electrochim Acta, 2019, 299 143-151

35 Cong R, Qiao S, Liu J, et al. Ultrahigh, ultrafast, and self-powered visible-near-infrared optical position-sensitive detector based on a CVD-prepared vertically standing few-layer $\mathrm{MoS}_{2} / \mathrm{Si}$ heterojunction. Adv Sci, 2018, 5: 1700502

36 Chen F, Wang Y, Su W, et al. Position-selective growth of 2D $\mathrm{WS}_{2-}$ based vertical heterostructures via a one-step CVD approach. J Phys Chem C, 2019, 123: 30519-30527

37 Jiang J, Li N, Zou J, et al. Synergistic additive-mediated CVD growth and chemical modification of 2D materials. Chem Soc Rev, 2019, 48: 4639-4654

38 Zhou J, Liu F, Lin J, et al. Large-area and high-quality 2D transition metal telluride. Adv Mater, 2017, 29: 1603471

39 Kum H, Lee D, Kong W, et al. Epitaxial growth and layer-transfer techniques for heterogeneous integration of materials for electronic and photonic devices. Nat Electron, 2019, 2: 439-450

$40 \mathrm{Xu} \mathrm{Y}$, Shi X, Zhang Y, et al. Epitaxial nucleation and lateral growth of high-crystalline black phosphorus films on silicon. Nat Commun, 2020, 11: 1330

41 Madani K, Rohatgi A, Min KH, et al. Comparison of passivation properties of plasma-assisted ALD and APCVD deposited $\mathrm{Al}_{2} \mathrm{O}_{3}$ with $\mathrm{SiN}_{x}$ capping. Sol Energy Mater Sol Cells, 2020, 218: 110718

42 Tanskanen JT, Bakke JR, Bent SF, et al. ALD growth characteristics of $\mathrm{ZnS}$ films deposited from organozinc and hydrogen sulfide precursors. Langmuir, 2010, 26: 11899-11906

43 Johnson RW, Hultqvist A, Bent SF. A brief review of atomic layer deposition: From fundamentals to applications. Mater Today, 2014 17: $236-246$

44 Baumgarten L, Szyjka T, Mittmann T, et al. Impact of vacancies and impurities on ferroelectricity in PVD- and ALD-grown $\mathrm{HfO}_{2}$ films. Appl Phys Lett, 2021, 118: 032903

45 Shakoury R, Arman A, Rezaee S, et al. Optical properties and morphology analysis of hexagonal $\mathrm{WO}_{3}$ thin films obtained by electron beam evaporation. J Mater Sci-Mater Electron, 2021, 32: 798-805

46 Cheng R, Bai J, Liao L, et al. High-frequency self-aligned graphene transistors with transferred gate stacks. Proc Natl Acad Sci U S A, 2012, 109: 11588-11592

47 Mueller T, Xia F, Avouris P. Graphene photodetectors for high-speed optical communications. Nat Photon, 2010, 4: 297-301

48 Lara-Avila S, Danilov A, Golubev D, et al. Towards quantum-limited coherent detection of terahertz waves in charge-neutral graphene. Nat Astron, 2019, 3: 983-988

49 Du X, Jiang W, Zhang Y, et al. Transparent and stretchable graphene electrode by intercalation doping for epidermal electrophysiology. ACS Appl Mater Interfaces, 2020, 12: 56361-56371

50 Ryzhii V, Ryzhii M. Graphene bilayer field-effect phototransistor for terahertz and infrared detection. Phys Rev B, 2009, 79: 245311

51 Ryzhii V, Ryzhii M, Ryabova N, et al. Graphene nanoribbon phototransistor: Proposal and analysis. Jpn J Appl Phys, 2009, 48: 04C144

52 Ryzhii V, Ponomarev DS, Ryzhii M, et al. Negative and positive terahertz and infrared photoconductivity in uncooled graphene. Opt Mater Express, 2019, 9: 585

53 Ryzhii V, Ryzhii M, Otsuji $\mathrm{T}$, et al. Heat capacity of nonequilibrium electron-hole plasma in graphene layers and graphene bilayers. Phys Rev B, 2021, 103: 245414 
54 Li X, Zhu M, Du M, et al. High detectivity graphene-silicon heterojunction photodetector. Small, 2016, 12: 595-601

55 Goykhman I, Sassi U, Desiatov B, et al. On-chip integrated, silicongraphene plasmonic schottky photodetector with high responsivity and avalanche photogain. Nano Lett, 2016, 16: 3005-3013

56 Selvi H, Hill EW, Parkinson P, et al. Graphene-silicon-on-insulator (GSOI) Schottky diode photodetectors. Nanoscale, 2018, 10: 1892618935

57 Iannaccone G, Fiori G, Macucci $M$ et al. Perspectives of graphene nanoelectronics: Probing technological options with modeling. In: 2009 IEEE International Electron Devices Meeting (IEDM 2009). Baltimore, MD, 2009. 1-4

58 Cao Y, Fatemi V, Demir A, et al. Correlated insulator behaviour at half-filling in magic-angle graphene superlattices. Nature, 2018, 556: 80-84

59 Cao Y, Fatemi V, Fang S, et al. Unconventional superconductivity in magic-angle graphene superlattices. Nature, 2018, 556: 43-50

60 Cao Y, Rodan-Legrain D, Rubies-Bigorda O, et al. Tunable correlated states and spin-polarized phases in twisted bilayer-bilayer graphene. Nature, 2020, 583: 215-220

61 Park JM, Cao Y, Watanabe K, et al. Tunable strongly coupled superconductivity in magic-angle twisted trilayer graphene. Nature, 2021, 590: 249-255

62 Zeng $\mathrm{LH}$, Wang $\mathrm{MZ}, \mathrm{Hu} \mathrm{H}$, et al. Monolayer graphene/germanium Schottky junction as high-performance self-driven infrared light photodetector. ACS Appl Mater Interfaces, 2013, 5: 9362-9366

63 Gusakova J, Wang X, Shiau LL, et al. Electronic properties of bulk and monolayer TMDs: Theoretical study within DFT framework (GVJ-2e Method). Phys Status Solidi A, 2017, 214: 1700218

64 Ermolaev GA, Stebunov YV, Vyshnevyy AA, et al. Broadband optical properties of monolayer and bulk $\mathrm{MoS}_{2}$. npj 2D Mater Appl, 2020, 4: 21

65 Mak KF, Lee C, Hone J, et al. Atomically thin $\mathrm{MoS}_{2}$ : A new direct-gap semiconductor. Phys Rev Lett, 2010, 105: 136805

66 Xie Y, Zhang B, Wang S, et al. Ultrabroadband $\mathrm{MoS}_{2}$ photodetector with spectral response from 445 to $2717 \mathrm{~nm}$. Adv Mater, 2017, 29: 1605972

67 Wang X, Wang P, Wang J, et al. Ultrasensitive and broadband $\mathrm{MoS}_{2}$ photodetector driven by ferroelectrics. Adv Mater, 2015, 27: 65756581

68 Xiao P, Mao J, Ding K, et al. Solution-processed 3D RGO-MoS 2 pyramid $\mathrm{Si}$ heterojunction for ultrahigh detectivity and ultra-broadband photodetection. Adv Mater, 2018, 30: 1801729

69 Nalwa HS. A review of molybdenum disulfide $\left(\mathrm{MoS}_{2}\right)$ based photodetectors: From ultra-broadband, self-powered to flexible devices. RSC Adv, 2020, 10: 30529-30602

70 Novais Antunes FP, Vaiss VS, Tavares SR, et al. Van der Waals interactions and the properties of graphite and $2 \mathrm{H}-, 3 \mathrm{R}-$ and $1 \mathrm{~T}-\mathrm{MoS}_{2}$ : A comparative study. Comput Mater Sci, 2018, 152: 146-150

71 Duerloo KAN, Li Y, Reed EJ. Structural phase transitions in twodimensional Mo- and $\mathrm{W}$-dichalcogenide monolayers. Nat Commun, 2014, 5: 4214

72 Jayabal $\mathrm{S}, \mathrm{Wu}$ J, Chen J, et al. Metallic $1 \mathrm{~T}-\mathrm{MoS}_{2}$ nanosheets and their composite materials: Preparation, properties and emerging applications. Mater Today Energy, 2018, 10: 264-279

73 Wang W, Zeng X, Warner JH, et al. Photoresponse-bias modulation of a high-performance $\mathrm{MoS}_{2}$ photodetector with a unique vertically stacked 2H-MoS $/ 1 \mathrm{~T} @ 2 \mathrm{H}-\mathrm{MoS}_{2}$ structure. ACS Appl Mater Interfaces, 2020, 12: 33325-33335

74 Strachan J, Masters AF, Maschmeyer T. 3R-MoS 2 in review: History, status, and outlook. ACS Appl Energy Mater, 2021, 4: 7405-7418

75 Irfan I, Golovynskyi S, Bosi M, et al. Enhancement of Raman scattering and exciton/trion photoluminescence of monolayer and fewlayer $\mathrm{MoS}_{2}$ by Ag nanoprisms and nanoparticles: Shape and size effects. J Phys Chem C, 2021, 125: 4119-4132

76 Shi J, Yu P, Liu F, et al. 3R $\mathrm{MoS}_{2}$ with broken inversion symmetry: A promising ultrathin nonlinear optical device. Adv Mater, 2017, 29: 1701486

77 Fang Y, Pan J, He J, et al. Structure re-determination and super- conductivity observation of bulk $1 \mathrm{~T} \mathrm{MoS}$. Angew Chem Int Ed, 2018 57: $1232-1235$

78 Wang X, Gong Y, Shi G, et al. Chemical vapor deposition growth of crystalline monolayer $\mathrm{MoSe}_{2}$. ACS Nano, 2014, 8: 5125-5131

79 Kumar N, Cui Q, Ceballos F, et al. Exciton diffusion in monolayer and bulk $\mathrm{MoSe}_{2}$. Nanoscale, 2014, 6: 4915-4919

80 Zhao X, Zhang L, Gai Q, et al. High-performance position-sensitive detector based on the lateral photovoltaic effect in $\mathrm{MoSe}_{2} / \mathrm{p}-\mathrm{Si}$ junctions. Appl Opt, 2019, 58: 5200-5205

81 Mao J, Yu Y, Wang L, et al. Ultrafast, broadband photodetector based on $\mathrm{MoSe}_{2} /$ silicon heterojunction with vertically standing layered structure using graphene as transparent electrode. Adv Sci, 2016, 3: 1600018

82 Ko PJ, Abderrahmane A, Kim N, et al. High-performance near-infrared photodetector based on nano-layered $\mathrm{MoSe}_{2}$. Semicond Sci Technol, 2017, 32: 065015

83 John JW, Dhyani V, Maity S, et al. Broadband infrared photodetector based on nanostructured $\mathrm{MoSe}_{2}-\mathrm{Si}$ heterojunction extended up to $2.5 \mu \mathrm{m}$ spectral range. Nanotechnology, 2020, 31: 455208

84 Kim HS, Patel M, Kim J, et al. Growth of wafer-scale standing layers of $\mathrm{WS}_{2}$ for self-biased high-speed UV-visible-NIR optoelectronic devices. ACS Appl Mater Interfaces, 2018, 10: 3964-3974

$85 \mathrm{Wu}$ E, Wu D, Jia C, et al. In situ fabrication of $2 \mathrm{D} \mathrm{WS} / \mathrm{Si}$ type-II heterojunction for self-powered broadband photodetector with response up to mid-infrared. ACS Photonics, 2019, 6: 565-572

86 Patel M, Pataniya PM, Late DJ, et al. Plasmon-enhanced photoresponse in $\mathrm{Ag}-\mathrm{WS}_{2} / \mathrm{Si}$ heterojunction. Appl Surf Sci, 2021, 538 148121

87 Octon T, Nagareddy V, Gracium M, et al. Photoconductivity of fewlayer $\mathrm{MoTe}_{2}$. The 7th International Conference on Metamaterials, Photonic Crystals and Plasmonics. Malaga, 2016

$88 \mathrm{Lu} \mathrm{Z,} \mathrm{Xu} \mathrm{Y,} \mathrm{Yu} \mathrm{Y,} \mathrm{et} \mathrm{al.} \mathrm{Ultrahigh} \mathrm{speed} \mathrm{and} \mathrm{broadband} \mathrm{few-layer}$ $\mathrm{MoTe}_{2} / \mathrm{Si}$ 2D-3D heterojunction-based photodiodes fabricated by pulsed laser deposition. Adv Funct Mater, 2020, 30: 1907951

89 Lin DY, Hsu HP, Liu GH, et al. Enhanced photoresponsivity of $2 \mathrm{H}-$ $\mathrm{MoTe}_{2}$ by inserting $1 \mathrm{~T}-\mathrm{MoTe}_{2}$ interlayer contact for photodetector applications. Crystals, 2021, 11: 964

90 Zheng Z, Zhang T, Yao J, et al. Flexible, transparent and ultrabroadband photodetector based on large-area $\mathrm{WSe}_{2}$ film for wearable devices. Nanotechnology, 2016, 27: 225501

91 Pataniya PM, Zankat CK, Tannarana M, et al. Photovoltaic activity of $\mathrm{WSe}_{2} / \mathrm{Si}$ hetero junction. Mater Res Bull, 2019, 120: 110602

92 Oyedele AD, Yang S, Liang L, et al. PdSe $e_{2}$ : Pentagonal two-dimensional layers with high air stability for electronics. J Am Chem Soc, 2017, 139: 14090-14097

93 Deng S, Li L, Zhang Y. Strain modulated electronic, mechanical, and optical properties of the monolayer $\mathrm{PdS}_{2}, \mathrm{PdSe}_{2}$, and $\mathrm{PtSe}_{2}$ for tunable devices. ACS Appl Nano Mater, 2018, 1: 1932-1939

94 Qin D, Yan P, Ding G, et al. Monolayer $\mathrm{PdSe}_{2}$ : A promising twodimensional thermoelectric material. Sci Rep, 2018, 8: 2764

95 Zeng LH, Wu D, Lin SH, et al. Controlled synthesis of 2D palladium diselenide for sensitive photodetector applications. Adv Funct Mater, 2019, 29: 1806878

96 Liang FX, Zhao XY, Jiang JJ, et al. Light confinement effect induced highly sensitive, self-driven near-infrared photodetector and image sensor based on multilayer $\mathrm{PdSe}_{2} /$ pyramid Si heterojunction. Small, 2019, 15: 1903831

97 Aftab S, Samiya M, Liao W, et al. Switching photodiodes based on

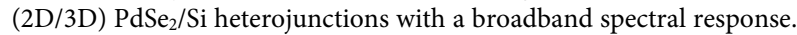
J Mater Chem C, 2021, 9: 3998-4007

98 Chung CC, Yeh $\mathrm{H}$, Wu PH, et al. Atomic-layer controlled interfacial band engineering at two-dimensional layered $\mathrm{PtSe}_{2} / \mathrm{Si}$ heterojunctions for efficient photoelectrochemical hydrogen production. ACS Nano, 2021, 15: 4627-4635

99 Shawkat MS, Chung HS, Dev D, et al. Two-dimensional/three-dimensional Schottky junction photovoltaic devices realized by the direct CVD growth of vdW 2D PtSe $e_{2}$ layers on silicon. ACS Appl Mater Interfaces, 2019, 11: 27251-27258

100 Liu F, Zheng S, He X, et al. Highly sensitive detection of polarized 
light using anisotropic 2D ReS 2 . Adv Funct Mater, 2016, 26: 11691177

101 Mukherjee B, Zulkefli A, Hayakawa R, et al. Enhanced quantum efficiency in vertical mixed-thickness $\mathrm{n}-\mathrm{ReS}_{2} / \mathrm{p}$-Si heterojunction photodiodes. ACS Photonics, 2019, 6: 2277-2286

102 Ahn J, Kyhm JH, Kang HK, et al. 2D $\mathrm{MoTe}_{2} / \mathrm{ReS}_{2}$ van der Waals heterostructure for high-performance and linear polarization-sensitive photodetector. ACS Photonics, 2021, 8: 2650-2658

103 Zeb J, Zhao X, Ullah S, et al. Tunable optoelectronic properties in multilayer $1 \mathrm{~T}-\mathrm{TiS}_{2}$ : The effects of strain and an external electric field. J Mater Sci, 2021, 56: 6891-6902

104 Yin J, Chen P, Lu M, et al. Cu-doped $\mathrm{CoS}_{2}$ polyhedrons with high catalytic activity and long-term stability. Sci China Mater, 2020, 63: 1337-1344

105 Dai C, Li B, Li J, et al. Controllable synthesis of $\mathrm{NiS}$ and $\mathrm{NiS}_{2}$ nanoplates by chemical vapor deposition. Nano Res, 2020, 13: 2506-2511

106 Ruan W, Chen Y, Tang S, et al. Evidence for quantum spin liquid behaviour in single-layer $1 \mathrm{~T}-\mathrm{TaSe}_{2}$ from scanning tunnelling microscopy. Nat Phys, 2021, 17: 1154-1161

107 Wang K, Guo Z, Li Y, et al. Few-layer $\mathrm{NbTe}_{2}$ nanosheets as substrates for surface-enhanced Raman scattering analysis. ACS Appl Nano Mater, 2020, 3: 11363-11371

108 Tannarana M, Solanki GK, Bhakhar SA, et al. 2D-SnSe $e_{2}$ nanosheet functionalized piezo-resistive flexible sensor for pressure and human breath monitoring. ACS Sustain Chem Eng, 2020, 8: 7741-7749

109 Li L, Yu Y, Ye GJ, et al. Black phosphorus field-effect transistors. Nat Nanotech, 2014, 9: 372-377

110 Yin Y, Cao R, Guo J, et al. High-speed and high-responsivity hybrid silicon/black-phosphorus waveguide photodetectors at $2 \mu \mathrm{m}$. Laser Photonics Rev, 2019, 13: 1900032

111 Huang L, Dong B, Guo X, et al. Waveguide-integrated black phosphorus photodetector for mid-infrared applications. ACS Nano, 2019, 13: 913-921

112 Yuan S, Shen C, Deng B, et al. Air-stable room-temperature midinfrared photodetectors based on hBN/black arsenic phosphorus/hBN heterostructures. Nano Lett, 2018, 18: 3172-3179

113 Zhang Y, Zhang F, Xu Y, et al. Self-healable black phosphorus photodetectors. Adv Funct Mater, 2019, 29: 1906610

114 Kao KH, Verhulst AS, Vandenberghe WG, et al. Direct and indirect band-to-band tunneling in germanium-based TFETs. IEEE Trans Electron Devices, 2012, 59: 292-301

115 Mahyavanshi RD, Kalita G, Ranade A, et al. Photovoltaic action with broadband photoresponsivity in germanium- $\mathrm{MoS}_{2}$ ultrathin heterojunction. IEEE Trans Electron Devices, 2018, 65: 4434-4440

116 Chen W, Liang R, Wang J, et al. Enhanced photoresponsivity and hole mobility of $\mathrm{MoTe}_{2}$ phototransistors by using an $\mathrm{Al}_{2} \mathrm{O}_{3}$ high- $\kappa$ gate dielectric. Sci Bull, 2018, 63: 997-1005

117 Chen W, Liang $\mathrm{R}$, Zhang S, et al. Ultrahigh sensitive near-infrared photodetectors based on $\mathrm{MoTe}_{2}$ /germanium heterostructure. Nano Res, 2020, 13: 127-132

118 Zhuo R, Zeng L, Yuan $\mathrm{H}$, et al. In-situ fabrication of $\mathrm{PtSe}_{2} / \mathrm{GaN}$ heterojunction for self-powered deep ultraviolet photodetector with ultrahigh current on/off ratio and detectivity. Nano Res, 2019, 12: 183189

119 Zhuo R, Wang Y, Wu D, et al. High-performance self-powered deep ultraviolet photodetector based on $\mathrm{MoS}_{2} / \mathrm{GaN}$ p-n heterojunction. J Mater Chem C, 2018, 6: 299-303

120 Liu X, Hu S, Lin Z, et al. High-performance $\mathrm{MoS}_{2}$ photodetectors prepared using a patterned gallium nitride substrate. ACS Appl Mater Interfaces, 2021, 13: 15820-15826

121 Nasr JR, Simonson N, Oberoi A, et al. Low-power and ultra-thin $\mathrm{MoS}_{2}$ photodetectors on glass. ACS Nano, 2020, 14: 15440-15449

$122 \mathrm{Xu} \mathrm{Z}$, Lin S, Li X, et al. Monolayer $\mathrm{MoS}_{2} / \mathrm{GaAs}$ heterostructure selfdriven photodetector with extremely high detectivity. Nano Energy, 2016, 23: 89-96
Acknowledgements This work was financially supported by the National Key Research and Development Program of China (2017YFA0207500), the National Natural Science Foundation of China (62125404), and the CAS JSPS Cooperative Research Project (GJHZ2021131).

Author contributions Original idea was conceived by Wei Z and Zhao X Manuscript was drafted by Zhou J and Xin K. All authors discussed and commented on the manuscript.

Conflict of interest The authors declare that they have no conflict of interest.

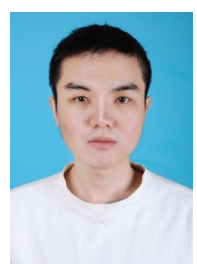

Jingshu Zhou received his BSc degree in microelectronic engineering from Nankai University in 2013. Currently, he is a $\mathrm{PhD}$ student under the supervision of Prof. Zhongming Wei at the Institute of Semiconductors, Chinese Academy of Sciences (IOS-CAS). His research interests focus on the preparation and photoelectric properties of 2D materials.

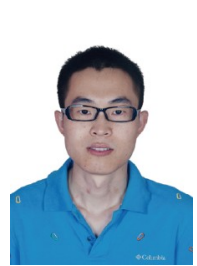

Xiangkai Zhao received his BSc degree in optoelectronic information science and engineering from Jilin University in 2003. Currently, he is working as an engineer at IOSCAS. His research interests focus on optoelectronic detection and optoelectronic system.

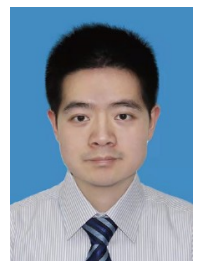

Zhongming Wei received his BS degree from Wuhan University (China) in 2005, and $\mathrm{PhD}$ degree from the Institute of Chemistry, CAS (ICCAS) in 2010 under the supervision of Prof. Daoben Zhu and Prof. Wei Xu. From August 2010 to January 2015, he worked as a postdoctoral fellow and then assistant professor in Prof. Thomas Bjørnholm's group at the University of Copenhagen, Denmark. Currently, he is working as a Professor at IOSCAS. His research interests include low-dimensional semiconductors and their optoelectronic devices.

\section{二维/三维硅基异质结光电应用研究进展}

$$
\text { 周劲澍 }{ }^{1,2} \text {, 辛凯耀 }{ }^{1} \text {, 赵向凯 } 3^{*} \text {, 李冬梅 }{ }^{3} \text {, 魏钟鸣 }{ }^{1 *} \text {, 夏建白 }{ }^{1}
$$

摘要 以硅为基础的半导体技术在过去几十年随着摩尔定律取得了很 大进展, 但是它也正接近自己的物理极限. 近年来, 二维材料因其独特 的结构和优越的性能, 被认为是突破传统硅基光电器件限制的契机. 本 文针对二维材料探测器与三维硅基系统混合集成的研究现状, 简述了 几种用于光电探测器的二维材料的基本特性. 随后, 总结了基于二维材 料的硅光子集成光电探测器的研究进展, 并对其器件结构和主要性能 进行了展述. 其后, 又补充介绍了结合其他传统材料为衬底的二维器 件. 最后, 我们对二维/三维混合硅基异质结构的发展前景进行了展望. 